TAIWANESE JOURNAL OF MATHEMATICS

Vol. 13, No. 1, pp. 91-135, February 2009

This paper is available online at http://www.tjm.nsysu.edu.tw/

\title{
BOUNDEDNESS OF SINGULAR INTEGRALS IN HARDY SPACES ON SPACES OF HOMOGENEOUS TYPE
}

\author{
Guoen Hu, Dachun Yang* and Yuan Zhou
}

\begin{abstract}
The authors first give a detailed proof on the coincidence between atomic Hardy spaces of Coifman and Weiss on a space of homogeneous type with those Hardy spaces on the same underlying space with the original distance replaced by the measure distance. Then the authors present some general criteria which guarantee the boundedness of considered linear operators from a Hardy space to some Lebesgue space or Hardy space, provided that it maps all atoms into uniformly bounded elements of that Lebesgue space or Hardy space. Third, the authors obtain the boundedness in Hardy spaces of singular integrals with kernels only having weak regularity by characterizing these Hardy spaces with a new kind of molecules, which is deeply related to the kernels of considered singular integrals. Finally, as an application, the authors obtain the boundedness in Hardy spaces of Monge-Ampère singular integral operators.
\end{abstract}

\section{INTRODUCTION}

The theory of Hardy spaces and singular integrals on Euclidean spaces $\mathbb{R}^{m}$ played an important role in analysis such as harmonic analysis and partial differential equations; see, for examples, [34, 17, 10, 28, 30, 32]. One of the most important applications of Hardy spaces is that they are good substitutes of Lebesgue spaces when $p \leq 1$. For example, when $p \leq 1$, it is well-known that Riesz transforms are not bounded on $L^{p}\left(\mathbb{R}^{m}\right)$, however, they are bounded on Hardy spaces $H^{p}\left(\mathbb{R}^{m}\right)$.

Accepted June 29, 2007.

Communicated by Yong-Sheng Han.

2000 Mathematics Subject Classification: 42B20, 42B30, 43A99.

Key words and phrases: Hardy space, Atom, Molecule, Space of homogeneous type, BMO, Lipschitz space, Singular integral, Monge-Ampère singular integral, Variable kernel, Unbounded model domains of polynomial type, Matrix dilation.

The first author is supported by National Natural Science Foundation of China (No. 10671210), and the second (corresponding) author was supported by National Science Foundation for Distinguished Young Scholars (No. 10425106) and NCET (No. 04-0142) of Ministry of Education of China.

*Corresponding author. 
To establish the boundedness of operators in Hardy spaces on $\mathbb{R}^{m}$, one usually has recourse to the atomic decomposition characterization (see $[9,22])$ or the molecular characterization (see [35]) of Hardy spaces, which means that a function or distribution in Hardy spaces can be represented as a linear combination of functions of an elementary form, namely, atom or molecule. Thus, the boundedness of linear operators in Hardy spaces can be deduced from their behavior on atoms or molecules in principle. However, recently, using a fact due to Y. Meyer (see [27] or [16, Section III 8.3]) that quasi-norms corresponding to finite and infinite atomic decompositions in Hardy spaces are not equivalent, Bownik in [3] gave a rather surprising example to indicate that an operator from a Hardy space $H^{p}\left(\mathbb{R}^{m}\right)$ with $p \in(0,1]$ into certain quasi-Banach space $\mathcal{B}$ mapping all atoms into uniformly bounded elements of $\mathcal{B}$ cannot guarantee that this operator extends to a bounded operator from $H^{p}\left(\mathbb{R}^{m}\right)$ to $\mathcal{B}$. We should point out that this phenomenon was essentially already observed by Y. Meyer in [26, p. 19].

Thus, a natural question appears, namely, what is the natural condition which can guarantee the boundedness of considered operators from a Hardy space to some quasi-Banach space $\mathcal{B}$, if it maps all atoms into uniformly bounded elements of $\mathcal{B}$ ? Yabuta in [36] found some very general sufficient and natural conditions in $\mathbb{R}^{m}$ for the boundedness of $T$ from $H^{p}\left(\mathbb{R}^{m}\right)$ with $p \in(0,1]$ to $L^{q}\left(\mathbb{R}^{m}\right)$ with $q \geq 1$ or $H^{q}\left(\mathbb{R}^{m}\right)$ with $q \in[p, 1]$. But, the case on the boundedness of $T$ from $H^{p}\left(\mathbb{R}^{m}\right)$ with $p \in(0,1]$ to $L^{q}\left(\mathbb{R}^{m}\right)$ with $q \in[p, 1)$ is still missing.

On the other hand, based on the atomic characterization of Hardy spaces in [9, 22], Coifman and Weiss [12] introduced the atomic Hardy spaces on a space of homogeneous type in [11], which is known to be a natural setting for the theory of Hardy spaces and singular integrals.

We first recall the definition of spaces of homogeneous type; see [11, 12]. Let $\mathcal{X}$ be a set. Endow $\mathcal{X}$ with a positive Borel regular measure $\mu$ and a quasi-metric $d$ satisfying that there exists $C_{1} \geq 1$ such that for all $x, y, z \in \mathcal{X}$,

$$
d(x, y) \leq C_{1}(d(x, z)+d(y, z)) .
$$

The triple $(\mathcal{X}, d, \mu)$ is said to be a space of homogeneous type in the sense of Coifman and Weiss ([11, 12]) if $\mu$ is doubling, namely, there exists $C_{2} \geq 1$ such that for all $x \in \mathcal{X}$ and $r>0$,

$$
\mu\left(B_{d}(x, 2 r)\right) \leq C_{2} \mu\left(B_{d}(x, r)\right),
$$

where $B_{d}(x, r)=\{y \in \mathcal{X}: d(x, y)<r\}$.

It is easy to see that the condition (1.2) is equivalent to that there exist constants $n>0$ and $C_{3} \geq 1$ such that for all $x \in \mathcal{X}, r>0$ and $\lambda>1$,

$$
\mu\left(B_{d}(x, \lambda r)\right) \leq C_{3} \lambda^{n} \mu\left(B_{d}(x, r)\right) .
$$


We remark that although all balls defined by $d$ satisfy the axioms of complete system of neighborhoods in $\mathcal{X}$, and therefore induce a (separated) topology in $\mathcal{X}$, the balls $B_{d}(x, r)$ for $x \in \mathcal{X}$ and $r>0$ need not to be open with respect to this topology. However, by Theorem 2 in [23], we know that there exists a quasi-metric $\widetilde{d}$ such that $\widetilde{d}$ is equivalent to $d$ and the balls corresponding to $\widetilde{d}$ are open in the topology induced by $\widetilde{d}$. Based on this, in what follows, we always assume that the balls corresponding to $d$ are open in the topology induced by $d$. Otherwise, we replace $d$ by $\widetilde{d}$, since all results in this paper are invariant for equivalent quasimetrics. Throughout this paper, we also assume that $\mu(\mathcal{X})=\infty$ and $\mu(\{x\})=0$ for all $x \in \mathcal{X}$.

Recall that the measure distance $\rho$, induced by the quasi-metric $d$ and the measure $\mu$, is defined by that for all $x, y \in \mathcal{X}$,

$$
\rho(x, y)=\inf \left\{\mu\left(B_{d}\right): B_{d} \text { is any ball containing } x \text { and } y\right\} ;
$$

see $[12,23]$. Macías and Segovia [23] proved that if the balls corresponding to $d$ are open in the topology induced by $d$, then $\rho$ is a quasi-metric where we denote by $C_{4}$ the corresponding constant in (1.1), the topologies on $\mathcal{X}$ induced by $d$ and $\rho$ coincide, and moreover, there exists $C_{5} \geq 1$ such that for all $x \in \mathcal{X}$ and $r>0$,

$$
C_{5}^{-1} r \leq \mu\left(B_{\rho}(x, r)\right) \leq C_{5} r
$$

see Theorem 3 in [23]. We conveniently mention that if $\mu$ and $\rho$ satisfy (1.4), then the triple $(\mathcal{X}, \rho, \mu)$ is called to be normal; see [23, p. 258]. In general, $\rho$ is not equivalent to $d$. We recall that the quasi-metric $\rho$ is said to be equivalent to the quasi-metric $d$ if there exists $C>0$ such that for all $x, y \in \mathcal{X}, C^{-1} d(x, y) \leq \rho(x, y) \leq C d(x, y)$. Macías and Segovia in [23, Theorem 2] proved that there exists a quasi-metric $\widetilde{\rho}$ on $\mathcal{X}$ which is equivalent to $\rho$ and satisfies that there exist constants $\theta \in(0,1)$ and $C>0$ such that for all $x, x^{\prime}, y \in \mathcal{X}$,

$$
\left|\widetilde{\rho}(x, y)-\widetilde{\rho}\left(x^{\prime}, y\right)\right| \leq C\left[\widetilde{\rho}\left(x, x^{\prime}\right)\right]^{\theta}\left[\widetilde{\rho}(x, y)+\widetilde{\rho}\left(x^{\prime}, y\right)\right]^{1-\theta} .
$$

Noting again that all the conclusions in this paper are invariant for equivalent quasimetrics, thus, when it is necessary, we may also assume that $\rho$ itself satisfies (1.5). In the sequel, $\theta$ is always taken to be the same as in (1.5).

Generally speaking, for two topologically equivalent spaces of homogeneous type, the corresponding Hardy spaces are not necessary to be equivalent; see, for example, [4, Theorem 10.5]. We recall that two quasi-Banach spaces $\mathcal{B}_{1}$ and $\mathcal{B}_{2}$ are said to be equivalent if they are equal as a set and their norms are equivalent. However, in Section 2 of this paper, we prove that atomic Hardy spaces of Coifman and Weiss on $(\mathcal{X}, d, \mu)$ are equivalent to those Hardy spaces on $(\mathcal{X}, \rho, \mu)$, which was mentioned in [12, p. 594] and [24, p. 271] without a proof. For the importance 
of Hardy spaces in applications and the convenience of the reader, we present a detailed proof of this fact by first establishing certain geometric measure relations between $(\mathcal{X}, d, \mu)$ and $(\mathcal{X}, \rho, \mu)$; see Theorem 2.1 and Proposition 2.1 below.

We point out that if $p \in(1 /(1+\theta), 1]$, Macías and Segovia [24] established a maximal function characterization for $H^{p}(\mathcal{X}, \rho, \mu)$, and Han [18] obtained a Lusinarea characterization for $H^{p}(\mathcal{X}, \rho, \mu)$ by using Coifman's approximations to the identity in [13].

Motivated by Yabuta [36], using the maximal function characterization for $H^{p}(\mathcal{X}, \rho, \mu)$ in [24], in Section 3, we generalize Yabuta's results on $\mathbb{R}^{m}$ to a space of homogeneous type $\mathcal{X}$ when $p \in(1 /(1+\theta), 1]$; see Theorem 3.1 below. Moreover, in Theorem 3.2 below, we also give certain sufficient conditions for the boundedness of $T$ from $H^{p}(\mathcal{X})$ to $L^{q}(\mathcal{X})$ with $p \in(1 /(1+\theta), 1)$ and $q \in[p, 1)$, which is new even on $\mathbb{R}^{m}$. Note that at the end-point case $p=1 /(1+\theta)$, the method in [36] is not valid; see Remark 3.1 (a). However, using some basic ideas of Y. Meyer in [26, Chapter 7] and Coifman's approximations to the identity in [13], we then obtain a weak and natural variant of Yabuta's result when $p=1 /(1+\theta)$ and also present certain sufficient condition for the boundedness of $T$ from $H^{p}(\mathcal{X})$ with $p \in[1 /(1+\theta), 1]$ to $H^{q}(\mathcal{X})$ with $q \in[1 /(1+\theta), 1]$ or $L^{q}(\mathcal{X})$ with $q \in[1 /(1+\theta), \infty)$; see Theorem 3.3 below.

Similarly to [29] on $\mathbb{R}^{m}$, we introduce the following classes of functions with weak regularity, which on $\mathbb{R}^{m}$ include functions having Dini's growth.

Definition 1.1. Let $\gamma \in[1, \infty]$ and $\eta=\left\{\eta_{j}\right\}_{j \in \mathbb{N}} \subset[0, \infty)$. A function $K$ defined on $\mathcal{X} \times \mathcal{X} \backslash\{(x, x): \quad x \in \mathcal{X}\}$ is said to be in $D_{\rho}(\gamma, \eta)$ if there exists $C_{K} \geq 2 C_{4}$ such that for all $x, y \in \mathcal{X}$ and $j \in \mathbb{N}$,

$$
\begin{aligned}
& \left\{\int_{R_{j}\left(B_{\rho}\left(x, C_{K} \rho(x, y)\right)\right)}|K(z, x)-K(z, y)|^{\gamma} d \mu(z)\right\}^{1 / \gamma} \\
& \quad \leq \eta_{j}\left[\mu\left(B_{\rho}\left(x, 2^{j} C_{K} \rho(x, y)\right)\right)\right]^{1 / \gamma-1},
\end{aligned}
$$

where and in what follows $R_{k}\left(B_{\rho}(x, r)\right)=\left\{y \in \mathcal{X}: 2^{k-1} r \leq \rho(x, y)<2^{k} r\right\}$ for $k \in \mathbb{N}$, and the usual modification is made when $\gamma=\infty$.

We give some typical examples of kernels on $\mathbb{R}^{m}$ satisfying Definition 1.1; see also [29] or Proposition 5.2 below. Let $K(x, y)=\Omega(x-y)|x-y|^{-m}$ for $x, y \in \mathbb{R}^{m}$ and $\Omega$ be homogeneous of degree zero. Let $\omega_{\gamma}$ be the $L^{\gamma}$-modulus of continuity of $\Omega$ over the unit sphere and for all $j \in \mathbb{N}, \eta_{j}=C\left[2^{-j / m}+\omega_{\gamma}\left(2^{-j / m}\right)\right]$ with a constant $C>0$. If $\int_{0}^{1} \omega_{\gamma}(t) t^{-1} d t<\infty$, which is often called the Dini condition, then $K \in$ $D_{\rho}(\gamma, \eta)$ with $\sum_{j \in \mathbb{N}} \eta_{j}<\infty$; If $\int_{0}^{1} \omega_{\gamma}(t)\left(\log t^{-1}\right) t^{-1} d t<\infty$, then $K \in D_{\rho}(\gamma, \eta)$ with $\sum_{j \in \mathbb{N}} j \eta_{j}<\infty$; If $m /(m+1)<p<1$ and $\int_{0}^{1}\left[\omega_{\gamma}(t)\right]^{p} t^{m(p-1)-1} d t<\infty$, then $K \in D_{\rho}(\gamma, \eta)$ with $\sum_{j \in \mathbb{N}}\left(\eta_{j}\right)^{p} 2^{j(1-p)}<\infty$. 
Another main purpose of this paper is to use the general criteria established in Section 3 to consider the boundedness from $H^{p}(\mathcal{X})$ to $L^{p}(\mathcal{X})$, from $H^{p}(\mathcal{X})$ to itself and from $H^{p}(\mathcal{X})$ to weak- $L^{p}(\mathcal{X})$ at the end-point case of singular integral operators $T$ with kernels as in Definition 1.1; see Theorem 4.1, Theorem 4.2 and Theorem 4.3 below.

To this end, we introduce the following kind of molecules, which is closely related to the kernels in Definition 1.1.

Definition 1.2. Let $0<p<q, p \leq 1 \leq q \leq \infty$ and $\eta=\left\{\eta_{k}\right\}_{k \in \mathbb{N}} \subset[0, \infty)$ satisfying

$$
\sum_{k=1}^{\infty} k \eta_{k}<\infty
$$

or when $p<1$,

$$
\sum_{k=1}^{\infty}\left(\eta_{k}\right)^{p} 2^{k(1-p)}<\infty .
$$

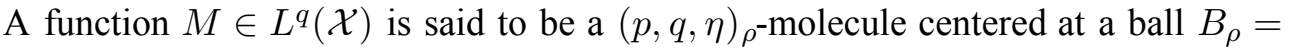
$B_{\rho}\left(x_{0}, r\right)$ for certain $x_{0} \in \mathcal{X}$ and $r>0$ if

(M1) $\|M\|_{L^{q}(\mathcal{X})} \leq\left[\mu\left(B_{\rho}\right)\right]^{1 / q-1 / p}$

(M2) for all $k \in \mathbb{N},\left\|M \chi_{R_{k}\left(B_{\rho}\right)}\right\|_{L^{q}(\mathcal{X})} \leq \eta_{k} 2^{k(1 / q-1)}\left[\mu\left(B_{\rho}\right)\right]^{1 / q-1 / p}$;

(M3) $\int_{\mathcal{X}} M(x) d \mu(x)=0$.

We establish the characterization for all Hardy spaces by this kind of molecules in Theorem 2.2 below and present its application in the study of the boundedness of operators in Corollary 3.1, which is a key tool of Section 4.

We should point out that if $\eta_{j}=2^{-j \epsilon}$ for $j \in \mathbb{N}$ and certain $\epsilon>0$, then molecules in Definition 1.2 coincide with the classical molecules; see for example $[12,35,25,15]$. Moreover, by Theorem 4 in [5], we know that the condition (1.7) with $p<1$ is sharp; see also Remark 2.2 below.

Finally, in Section 5 of this paper, we present an application of Theorem 4.1 through Theorem 4.3 to Monge-Ampere singular integral operators introduced by Caffarelli and Gutiérrez in [8].

We now make some conventions. Throughout this paper, we denote by $B_{d}$ and $B_{\rho}$ the balls induced by quasi-metrics $d$ and $\rho$, respectively. For all $x, y \in \mathcal{X}$, set $V(x, y)=\mu\left(B_{d}(x, d(x, y))\right)$. From (1.2), it is easy to deduce that $V(x, y) \sim$ $V(y, x)$. For any ball $B_{\rho}\left(x_{0}, r\right)$ with $x_{0} \in \mathcal{X}$ and $r>0$, write $R_{0}\left(B_{\rho}\left(x_{0}, r\right)\right)=$ $B_{\rho}\left(x_{0}, r\right)$ and $R_{k}\left(B_{\rho}\left(x_{0}, r\right)\right)=\left\{y \in \mathcal{X}: 2^{k-1} r \leq \rho\left(x_{0}, y\right)<2^{k} r\right\}$ with $k \in \mathbb{N}$, where 
$\mathbb{N}=\{1,2, \cdots\}$. We always denote by $C$ a positive constant that is independent of the main parameters involved but whose value may differ from line to line, and $f \lesssim g$ means $f \leq C g$. If $f \lesssim g \lesssim f$, we then write $f \sim g$. Constants with subscripts, such as $C_{1}$, do not change in different occurrences. We fix $N>1$ large enough such that $\left(C_{5}\right)^{-1} N-C_{5} \geq 1$. Without loss of generality, we may assume that $N=2$.

\section{Hardy Spaces on Spaces of Homogeneous Type}

In this section, for general spaces of homogeneous type, we first verify that the atomic Hardy space on $(\mathcal{X}, d, \mu)$ is equivalent to the atomic Hardy space on $(\mathcal{X}, \rho, \mu)$. We then establish a new characterization for these atomic Hardy spaces by using the molecules in Definition 1.2.

We begin with the definition of atomic Hardy spaces on $(\mathcal{X}, d, \mu)$ in [12]. To this end, we first recall the definitions of Lipschitz spaces, the space of functions with bounded mean oscillation and atoms; see [12].

Definition 2.1. Let $\alpha>0$. A function $f$ is said to be in $\operatorname{Lip}_{d}(\alpha)$ if there exists $C \geq 0$ such that for all $x, y \in \mathcal{X}$ and all balls $B_{d}$ containing $x$ and $y$,

$$
|f(x)-f(y)| \leq C\left[\mu\left(B_{d}\right)\right]^{\alpha} .
$$

The minimal constant $C$ in (2.1) is defined to be the $\operatorname{Lip}_{d}(\alpha)$ norm of $f$ and denoted by $\|f\|_{\operatorname{Lip}_{d}(\alpha)}$.

Definition 2.2. Let $1 \leq q<\infty$. A function $f$ is said to be in $\operatorname{BMO}^{q}(\mathcal{X}, d, \mu)$ if there exists $C \geq 0$ such that for all balls $B_{d} \subset \mathcal{X}$,

$$
\left\{\frac{1}{\mu\left(B_{d}\right)} \int_{B_{d}}\left|f(x)-f_{B_{d}}\right|^{q} d \mu(x)\right\}^{1 / q} \leq C,
$$

where and in what follows, $f_{B_{d}}=\frac{1}{\mu\left(B_{d}\right)} \int_{B_{d}} f(y) d \mu(y)$. The minimal constant $C$ in (2.2) is defined to be the $\mathrm{BMO}^{q}(\mathcal{X}, d, \mu)$ norm of $f$ and denoted by $\|f\|_{\mathrm{BMO}^{q}(\mathcal{X}, d, \mu)}$.

Denote $\operatorname{BMO}^{1}(\mathcal{X}, d, \mu)$ simply by $\operatorname{BMO}(\mathcal{X}, d, \mu)$. It is well-known that for $1<q<\infty, \operatorname{BMO}(\mathcal{X}, d, \mu)=\operatorname{BMO}^{q}(\mathcal{X}, d, \mu)$ with equivalent norms; see [12].

Definition 2.3. Let $0<p<q$ and $p \leq 1 \leq q \leq \infty$. A function $a$ is called to be a $(p, q)_{d}$-atom if
(A1) $\operatorname{supp} a \subset B_{d}=B_{d}(x, r)$ for certain $x \in \mathcal{X}$ and $r>0$;
(A2) $\|a\|_{L^{q}(\mathcal{X})} \leq\left[\mu\left(B_{d}\right)\right]^{1 / q-1 / p}$;
(A3) $\int_{\mathcal{X}} a(x) d \mu(x)=0$. 
Now we state the definition of atomic Hardy spaces. For $\alpha>0$, let $\left(\operatorname{Lip}_{d}(\alpha)\right)^{*}$ be the dual space of $\operatorname{Lip}_{d}(\alpha)$.

Definition 2.4. Let $0<p<q$ and $p \leq 1 \leq q \leq \infty$. A function $f \in$ $L^{1}(\mathcal{X})$ or a linear functional $f \in\left(\operatorname{Lip}_{d}(1 / p-1)\right)^{*}$ when $p<1$ is said to be in $H^{1, q}(\mathcal{X}, d, \mu)$ or in $H^{p, q}(\mathcal{X}, d, \mu)$ when $p<1$ if there exist $(p, q)_{d}$-atoms $\left\{a_{j}\right\}_{j=1}^{\infty}$ and $\left\{\lambda_{j}\right\}_{j=1}^{\infty} \subset \mathbb{C}$ such that $f=\sum_{j=1}^{\infty} \lambda_{j} a_{j}$, which converges in $L^{1}(\mathcal{X})$ when $p=1$ or in $\left(\operatorname{Lip}_{d}(1 / p-1)\right)^{*}$ when $p<1$, and $\sum_{j=1}^{\infty}\left|\lambda_{j}\right|^{p}<\infty$. Moreover, the norm of $f$ in $H^{p, q}(\mathcal{X}, d, \mu)$, denoted by $\|f\|_{H^{p, q}(\mathcal{X}, d, \mu)}$, is defined as $\inf \left\{\left(\sum_{j=1}^{\infty}\left|\lambda_{j}\right|^{p}\right)^{1 / p}\right\}$, where the infimum is taken over all the above decompositions of $f$.

Coifman and Weiss proved that $H^{p, q}(\mathcal{X}, d, \mu)=H^{p, \infty}(\mathcal{X}, d, \mu)$ for $0<p<q$ and $p \leq 1 \leq q \leq \infty,\left(H^{1, q}(\mathcal{X}, d, \mu)\right)^{*}=\operatorname{BMO}(\mathcal{X}, d, \mu)$ for $1<q \leq \infty$, and $\left(H^{p, q}(\mathcal{X}, d, \mu)\right)^{*}=\operatorname{Lip}_{d}(1 / p-1)$ for $0<p<1 \leq q \leq \infty$; see Theorem A and Theorem $\mathrm{B}$ in [12]. Therefore, in what follows, we denote $H^{p, q}(\mathcal{X}, d, \mu)$ simply by $H^{p}(\mathcal{X}, d, \mu)$.

If we replace $d$ by $\rho$ in Definition 2.1 through Definition 2.4, we then obtain the space $\operatorname{Lip}_{\rho}(\alpha), \operatorname{BMO}^{q}(\mathcal{X}, \rho, \mu),(p, q)_{\rho}$-atoms and atomic Hardy spaces $H^{p, q}(\mathcal{X}, \rho, \mu)$. All the conclusions stated above still hold for $H^{p, q}(\mathcal{X}, \rho, \mu), \mathrm{BMO}^{q}$ $(\mathcal{X}, \rho, \mu)$ and $\operatorname{Lip}_{\rho}(1 / p-1)$. Thus, in what follows, we denote $H^{p, q}(\mathcal{X}, \rho, \mu)$ simply by $H^{p}(\mathcal{X}, \rho, \mu)$.

\section{Remark 2.1.}

(a) From Definition 1.2 and Definition 2.3, it is easy to see that if $a$ is a $(p, q)_{\rho^{-}}$ atom supported in a ball $B_{\rho}$, then $a$ is a $(p, q, \eta) \rho_{\rho}$-molecule centered at the same ball $B_{\rho}$. Conversely, if $\eta_{k}=0$ for all $k \in \mathbb{N}$, then a $(p, q, \eta)_{\rho}$-molecule is just a $(p, q)$-atom. Moreover, by Definition 1.2, it is easy to see that the condition (1.6) or (1.7) implies that $\eta \in \ell^{1}$, and if $q_{1}<q_{2}$ and $M$ is a $\left(p, q_{2}, \eta\right)_{\rho}$-molecule, then there exists a constant $C>0$ independent of $M$ such that $\frac{1}{C} M$ is a $\left(p, q_{1}, \eta\right)_{\rho}$-molecule.

(b) By Theorem 5 in [23], Macías and Segovia proved that for $\alpha>0, f \in$ $\operatorname{Lip}_{d}(\alpha)$ if and only if there exists a constant $C>0$ such that for all $x, y \in$ $\mathcal{X}$

$$
|f(x)-f(y)| \leq C[\rho(x, y)]^{\alpha} .
$$

The minimum $C$ satisfying (2.3) is just $\|f\|_{\operatorname{Lip}_{d}(\alpha)}$. This result and (1.4) further indicate that for $\alpha>0, \operatorname{Lip}_{d}(\alpha)=\operatorname{Lip}_{\rho}(\alpha)$ with equivalent norms. In the sequel, we identify $\operatorname{Lip}_{d}(\alpha)$ with $\operatorname{Lip}_{\rho}(\alpha)$, and denote it simply by $\operatorname{Lip}(\alpha)$. 
(c) Although there exist different definitions of Hardy spaces on $(\mathcal{X}, \rho, \mu)$ according to $[12,24,18,19]$, we point out that they are essentially same. In fact, Han [18] proved that Hardy spaces in [18] coincide with those in [24]; see also [19]. If $p<1$, the equivalence between Hardy spaces in [12] and those in [24] is obvious; while if $p=1$, the coincidence between the Hardy space in [18] and that in [12] was presented in [19].

We can now state the main results of this section as follows.

Theorem 2.1. Let $0<p<q$ and $p \leq 1 \leq q \leq \infty$. Then there exists $a$ constant $C>0$ such that the function a is $a(p, q) d^{-a t o m}$ if and only if $\frac{1}{C} a$ is

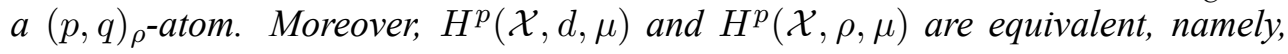
$H^{p}(\mathcal{X}, d, \mu)=H^{p}(\mathcal{X}, \rho, \mu)$ with equivalent norms.

The proof of Theorem 2.1 is given in Subsection 2.1 by first clarifying certain geometric measure relations between $(\mathcal{X}, d, \mu)$ and $(\mathcal{X}, \rho, \mu)$; see Proposition 2.1 below.

Theorem 2.2. Let $0<p<q, p \leq 1 \leq q \leq \infty$ and $\eta=\left\{\eta_{k}\right\}_{k \in \mathbb{N}} \subset[0, \infty)$ satisfying (1.6) or (1.7). Then there exists a constant $C>0$ such that for any $(p, q, \eta)_{\rho}$-molecule $M, M \in H^{p}(\mathcal{X}, \rho, \mu)$ and $\|M\|_{H^{p}(\mathcal{X}, \rho, \mu)} \leq C$. Moreover, $f \in H^{p}(\mathcal{X}, \rho, \mu)$ if and only if there exist $\left\{\lambda_{j}\right\}_{j=1}^{\infty} \subset \mathbb{C}$ and $(p, q, \eta)_{\rho^{-}}$ molecules $\left\{M_{j}\right\}_{j=1}^{\infty}$ such that $f=\sum_{j=1}^{\infty} \lambda_{j} M_{j}$, which converges in $L^{1}(\mathcal{X})$ when $p=1$ or in $(\operatorname{Lip}(1 / p-1))^{*}$ when $p<1$, and $\sum_{j=1}^{\infty}\left|\lambda_{j}\right|^{p}<\infty$. Furthermore, $\|f\|_{H^{p}(\mathcal{X}, \rho, \mu)} \sim \inf \left\{\left(\sum_{j=1}^{\infty}\left|\lambda_{j}\right|^{p}\right)^{1 / p}\right\}$, where the infimum is taken over all the above decompositions of $f$.

The proof of Theorem 2.2 is presented in Subsection 2.2. To this end, in Subsection 2.2, we first establish some basic properties of molecules in Definition 1.2; see Proposition 2.2 below.

\section{Remark 2.2.}

(a) By Theorem 2.1 and the duality theorem, we obtain $\operatorname{BMO}(\mathcal{X}, d, \mu)=\operatorname{BMO}$ $(\mathcal{X}, \rho, \mu)$ with equivalent norms. In what follows, we identify $H^{p}(\mathcal{X}, \rho, \mu)$ with $H^{p}(\mathcal{X}, d, \mu)$, and $\operatorname{BMO}(\mathcal{X}, d, \mu)$ with $\operatorname{BMO}(\mathcal{X}, \rho, \mu)$. Moreover, we denote them, respectively, simply by $H^{p}(\mathcal{X})$ and $\operatorname{BMO}(\mathcal{X})$.

(b) If $\eta_{j}=2^{-j \epsilon}$ for $j \in \mathbb{N}$ and certain $\epsilon>0$, then for $p \in(1 /(1+\epsilon), 1]$, the $(p, q, \eta)_{\rho}$-molecule in Definition 1.2 coincide with the classical one, and thus the molecular characterization for $H^{p}(\mathcal{X})$ established in Theorem 2.2 is an essential improvement of the known results; see, for example, [12, 35, 25, 15]. 
(c) By Theorem 4 in [5], when $p<1$, Theorem 2.2 is sharp in the sense that for any positive sequence $\left\{\epsilon_{j}\right\}_{j \in \mathbb{N}}$, if $\lim _{j \rightarrow \infty} \epsilon_{j}=0$ and $\eta$ satisfies that $\sum_{j \in \mathbb{N}}\left(\eta_{j}\right)^{p} 2^{j(1-p)} \epsilon_{j}<\infty$, then there exist $q \in[1, \infty)$ and $M$ satisfies (M1) through (M3) such that $M \notin H^{p}(\mathcal{X})$.

\subsection{Proof of Theorem 2.1}

We begin with some basic geometric measure properties of $\mathcal{X}$.

Proposition 2.1. There exists $C_{6}>0$ such that for any $x_{0} \in \mathcal{X}$ and $r_{0}>0$, there exists $\widetilde{r}_{0} \in(0, \infty)$, which may depend on $x_{0}$ and $r_{0}$, satisfying that $B_{\rho}\left(x_{0}, r_{0}\right) \subset B_{d}\left(x_{0}, \widetilde{r}_{0}\right)$ and $\mu\left(B_{d}\left(x_{0}, \widetilde{r}_{0}\right)\right) \leq C_{6} r_{0}$. Moreover, $\widetilde{r}_{0}$ is increasing on $r_{0}$, namely, if $\lambda>1$, then $\widetilde{r}_{0} \leq \widetilde{\lambda r_{0}}$.

Proof. Let

$$
r_{0}^{*}=\inf \left\{r>0: B_{\rho}\left(x_{0}, r_{0}\right) \subset B_{d}\left(x_{0}, r\right)\right\} .
$$

We first claim that $r_{0}^{*} \in(0, \infty)$. Since $r_{0}>0$ and $\mu\left(B_{\rho}\left(x_{0}, r_{0}\right)\right) \sim r_{0}$ by (1.4), then $\mu\left(B_{\rho}\left(x_{0}, r_{0}\right)\right)>0$. From this and $\mu(\{x\})=0$ together with the countable additivity of $\mu$, it is easy to deduce that $r_{0}^{*}>0$. If $r_{0}^{*}=\infty$, then for each $j \in \mathbb{N}$, there exists $y_{j} \in B_{\rho}\left(x_{0}, r_{0}\right) \backslash B_{d}\left(x_{0}, j\right)$. Thus $d\left(x_{0}, y_{j}\right) \geq j$ and $\rho\left(x_{0}, y_{j}\right)<r_{0}$. By $\rho\left(x_{0}, y_{j}\right)<r_{0}$ and the definition of $\rho$, there exists ball $B_{d}\left(x_{j}, r_{j}\right)$ containing $x_{0}$ and $y_{j}$ such that $\mu\left(B_{d}\left(x_{j}, r_{j}\right)\right)<r_{0}$. Therefore, by (1.1), $j \leq 2 C_{1} r_{j}$, which together with (1.1) again indicates $B_{d}\left(x_{0}, j\right) \subset B_{d}\left(x_{j}, 3\left(C_{1}\right)^{2} r_{j}\right)$. Hence, by (1.2), we have

$$
\mu\left(B_{d}\left(x_{0}, j\right)\right) \lesssim \mu\left(B_{d}\left(x_{j}, r_{j}\right)\right) \lesssim r_{0}
$$

On the other hand, by $\mu(\mathcal{X})=\infty$ and $\mathcal{X}=\cup_{j \in \mathbb{N}} B_{d}\left(x_{0}, j\right)$, we have $\mu\left(B_{d}\left(x_{0}, j\right)\right) \rightarrow$ $\infty$ as $j \rightarrow \infty$, which contradicts with (2.5). This verifies our claim.

Now we verify $\mu\left(B_{d}\left(x_{0}, 2 r_{0}^{*}\right)\right) \lesssim r_{0}$. In fact, by (2.4) and $r_{0}^{*} \in(0, \infty)$, we have $B_{\rho}\left(x_{0}, r_{0}\right) \backslash B_{d}\left(x_{0}, r_{0}^{*} / 2\right) \neq \emptyset$. Let $y_{0} \in B_{\rho}\left(x_{0}, r_{0}\right) \backslash B_{d}\left(x_{0}, r_{0}^{*} / 2\right)$. Then by $\rho\left(x_{0}, y_{0}\right)<r_{0}$ and the definition of $\rho$, there exists a ball $B_{d}\left(x^{\prime}, r^{\prime}\right)$ containing $x_{0}$ and $y_{0}$ such that

$$
\rho\left(x_{0}, y_{0}\right)<\mu\left(B_{d}\left(x^{\prime}, r^{\prime}\right)\right)<r_{0} .
$$

Moreover, by (1.1) and $r_{0}^{*} \leq 2 d\left(x_{0}, y_{0}\right)$ together with $d\left(x_{0}, x^{\prime}\right)<r^{\prime}$ and $d\left(y_{0}, x^{\prime}\right)<$ $r^{\prime}$, we have $B_{d}\left(x_{0}, 2 r_{0}^{*}\right) \subset B_{d}\left(x^{\prime}, 9\left(C_{1}\right)^{2} r^{\prime}\right)$, which via (1.2) and (2.6) yields that $\mu\left(B_{d}\left(x_{0}, 2 r_{0}^{*}\right)\right) \lesssim \mu\left(B_{d}\left(x^{\prime}, r^{\prime}\right)\right) \lesssim r_{0}$. Taking $\widetilde{r}_{0}=2 r_{0}^{*}$ gives us the first conclusion of Proposition 2.1. 
Obviously, if $\lambda>1, r_{0}^{*} \leq\left(\lambda r_{0}\right)^{*}$. This observation together with $\widetilde{r}_{0}=2 r_{0}^{*}$ and $\widetilde{\lambda r_{0}}=2\left(\lambda r_{0}\right)^{*}$ indicates that $\widetilde{r}_{0} \leq \widetilde{\lambda r_{0}}$, which completes the proof of Proposition 2.1.

Remark 2.3. By the assumption that $B_{d}(x, r)$ for all $x \in \mathcal{X}$ and $r>0$ is open and the definition of $\rho$, it is easy to see that for all $x \in \mathcal{X}$ and $r>0$, $B_{d}(x, r) \subset B_{\rho}\left(x, \mu\left(B_{d}(x, r)\right)\right)$, which together with Proposition 2.1 gives that for all $x \in \mathcal{X}$ and $r>0, B_{\rho}(x, r) \subset B_{d}(x, \widetilde{r}) \subset B_{\rho}\left(x, C_{6} r\right)$, and $B_{d}(x, r) \subset$ $B_{\rho}\left(x, r_{0}\right) \subset B_{d}\left(x, \widetilde{r_{0}}\right) \subset B_{\rho}\left(x, C_{6} r_{0}\right)$, where $r_{0}=\mu\left(B_{d}(x, r)\right)$. These relations indicate certain kind of "equivalence" between $d$ and $\rho$. We recall that $\rho$ is not necessarily equivalent to $d$.

From Proposition 2.1, we deduce the following conclusion, which is used in Section 4.1.

Corollary 2.1. There exists a constant $C_{7}>\left[C_{6} C_{5} C_{3}\right]^{1 / n}$ such that for all $x_{0} \in \mathcal{X}, r_{0}>0$ and $\lambda>1, \lambda^{1 / n} \widetilde{r}_{0} \leq C_{7} \widetilde{\lambda r_{0}}$, where $\widetilde{r}_{0}$ and $\widetilde{\lambda r_{0}}$ are the same as in Proposition 2.1.

Proof. Let $C_{7}>\left[C_{6} C_{5} C_{3}\right]^{1 / n}$. If $\lambda^{1 / n} \leq C_{7}$, since $\widetilde{r}_{0}$ is increasing on $r_{0}$, by Proposition 2.1, we immediately obtain the desired conclusion. Suppose now $\lambda^{1 / n}>C_{7}$. If $\lambda^{1 / n} \widetilde{r}_{0}>C_{7} \widetilde{\lambda r_{0}}$, then by Proposition 2.1, (1.4) and (1.3), we obtain

$$
\begin{aligned}
\mu\left(B_{d}\left(x_{0},\left(C_{7}\right)^{-1} \lambda^{1 / n} \widetilde{r}_{0}\right)\right) & \leq\left(C_{7}\right)^{-n} C_{3} \lambda \mu\left(B_{d}\left(x_{0}, \widetilde{r}_{0}\right)\right) \leq\left(C_{7}\right)^{-n} C_{3} C_{6} \lambda r_{0} \\
& \leq\left(C_{7}\right)^{-n} C_{3} C_{5} C_{6} \mu\left(B_{\rho}\left(x_{0}, \lambda r_{0}\right)\right)<\mu\left(B_{d}\left(x_{0}, \widetilde{\lambda r_{0}}\right)\right),
\end{aligned}
$$

which is a contradiction. Thus we have $\lambda^{1 / n} \widetilde{r}_{0} \leq C_{7} \widetilde{\lambda r_{0}}$, which completes the proof of Corollary 2.1.

The following conclusion is used in Subsection 2.2, we state it here. Recall that for $x, y \in \mathcal{X}, V(x, y)=\mu\left(B_{d}(x, d(x, y))\right)$.

Lemma 2.1. There exists a constant $C>0$ such that for all $x, y \in \mathcal{X}$, $C^{-1} \rho(x, y) \leq V(x, y) \leq C \rho(x, y)$.

Proof. Let $x, y \in \mathcal{X}$. From the definition of $\rho$ and (1.2), it follows that $\rho(x, y) \leq \mu\left(B_{d}(x, 2 d(x, y))\right) \lesssim V(x, y)$.

On the other hand, if $\rho(x, y)=0$, then $x=y$ and $V(x, y)=0$, which is the desired conclusion. We now suppose $\rho(x, y)>0$. Then, by the definition of $\rho$, there exists a ball $B_{d}=B_{d}\left(x_{0}, r\right)$ containing $x$ and $y$ such that $\mu\left(B_{d}\right) \leq 2 \rho(x, y)$, which together with (1.1) yields that $B_{d}(x, 2 d(x, y)) \subset B_{d}\left(x_{0}, 3 C_{1}^{2} r\right)$. From this, 
$\mu\left(B_{d}\right) \lesssim \rho(x, y)$ and (1.2), it follows that $V(x, y) \lesssim \rho(x, y)$, which completes the proof of Lemma 2.1.

We now turn to the proof of Theorem 2.1.

Proof of Theorem 2.1. Let $a$ be a $(p, q)_{d}$-atom supported in $B_{d}=B_{d}\left(x_{0}, r_{0}\right)$ for some $x_{0} \in \mathcal{X}$ and $r_{0}>0$. Then for all $y \in B_{d}$, by the definition of $\rho$, we have $\rho\left(x_{0}, y\right)<2 \mu\left(B_{d}\right)$, hence $y \in B_{\rho}\left(x_{0}, 2 \mu\left(B_{d}\right)\right)$, which implies that

$$
\operatorname{supp} a \subset B_{d} \subset B_{\rho}\left(x_{0}, 2 \mu\left(B_{d}\right)\right) .
$$

Moreover, by (1.4), we have $\mu\left(B_{\rho}\left(x_{0}, 2 \mu\left(B_{d}\right)\right)\right) \sim \mu\left(B_{d}\right)$, which together with (2.7), (A2) and (A3) indicates that there exists a constant $C>0$, which is independent of $a$, such that $\frac{1}{C} a$ is a $(p, q)_{\rho}$-atom.

On the other hand, let $a$ be a $(p, q)$-atom supported in $B_{\rho}=B_{\rho}\left(x_{0}, r_{0}\right)$ for some $x_{0} \in \mathcal{X}$ and $r_{0}>0$. By Proposition 2.1, there exists $\widetilde{r}_{0} \in(0, \infty)$ such that $\operatorname{supp} a \subset B_{\rho} \subset B_{d}\left(x_{0}, \widetilde{r}_{0}\right)$ and $\mu\left(B_{d}\left(x_{0}, \widetilde{r}_{0}\right)\right) \lesssim r_{0}$. Thus, by (A2) and (1.4), we have $\|a\|_{L^{q}(\mathcal{X})} \lesssim\left[\mu\left(B_{d}\left(x_{0}, \widetilde{r}_{0}\right)\right)\right]^{1 / q-1 / p}$, which indicates that there exists a constant $C>0$, independent of $a$, such that $\frac{1}{C} a$ is a $(p, q)_{d}$-atom. This proves the first conclusion of Theorem 2.1.

The second conclusion of Theorem 2.1 follows immediately from the definitions of Hardy spaces $H^{p}(\mathcal{X}, d, \mu)$ and $H^{p}(\mathcal{X}, \rho, \mu)$ together with Remark $2.1(\mathrm{~b})$, which completes the proof of Theorem 2.1.

\subsection{Proof of Theorem 2.2}

We begin with some properties of molecules in Definition 1.2.

Proposition 2.2. Let $0<p<q, p \leq 1 \leq q \leq \infty$ and $\eta=\left\{\eta_{k}\right\}_{k \in \mathbb{N}} \subset[0, \infty)$ satisfying (1.6) or (1.7). Let $M$ be a $(p, q, \eta)$-molecule centered at ball $B_{\rho}$. Then there exists a constant $C>0$ independent of $M$ such that

(i) $M \in L^{1}(\mathcal{X})$ and $\|M\|_{L^{1}(\mathcal{X})} \leq C\left[\mu\left(B_{\rho}\right)\right]^{1-1 / p}$;

(ii) $M \in L^{p}(\mathcal{X})$ and $\|M\|_{L^{p}(\mathcal{X})} \leq C$;

(iii) when $p<1, M \in(\operatorname{Lip}(1 / p-1))^{*}$ and $\|M\|_{(\operatorname{Lip}(1 / p-1))^{*}} \leq C$. Moreover, for all $f \in \operatorname{Lip}(1 / p-1),\langle M, f\rangle=\int_{\mathcal{X}} M(x) f(x) d \mu(x)$, where and in the sequel, $\langle\cdot, \cdot\rangle$ denotes the dual pair between $(\operatorname{Lip}(1 / p-1)) *$ and $\operatorname{Lip}(1 / p-1)$.

Proof. Let $M$ be a $(p, q, \eta)_{\rho}$-molecule centered at $B_{\rho}=B_{\rho}\left(x_{0}, r\right)$ for certain $x_{0} \in \mathcal{X}$ and $r>0$. Let $\eta_{0}=1$. For all $k \in \mathbb{N} \cup\{0\}$, by the Hölder inequality, (M1), (M2) and (1.4), we obtain

$$
\int_{R_{k}\left(B_{\rho}\right)}|M(x)| d \mu(x) \lesssim \eta_{k}\left[\mu\left(B_{\rho}\right)\right]^{1-1 / p}
$$


from which and Remark 2.1 (a), it follows that

$$
\|M\|_{L^{1}(\mathcal{X})} \leq \sum_{k=0}^{\infty} \int_{R_{k}\left(B_{\rho}\right)}|M(x)| d \mu(x) \lesssim \sum_{k=0}^{\infty} \eta_{k}\left[\mu\left(B_{\rho}\right)\right]^{1-1 / p} \lesssim\left[\mu\left(B_{\rho}\right)\right]^{1-1 / p} .
$$

Thus, (i) holds.

To verify (ii), by the Hölder inequality, (M1), (M2) and (1.4), we also deduce that

$$
\begin{aligned}
& \int_{R_{k}\left(B_{\rho}\right)}|M(x)|^{p} d \mu(x) \\
& \quad \leq\left[\mu\left(R_{k}\left(B_{\rho}\right)\right)\right]^{1-p / q}\left\{\int_{R_{k}\left(B_{\rho}\right)}|M(x)|^{q} d \mu(x)\right\}^{p / q} \lesssim 2^{k(1-p)}\left(\eta_{k}\right)^{p}
\end{aligned}
$$

for $k \in \mathbb{N} \cup\{0\}$, which together with (1.6) or (1.7) when $p<1$ indicates

$$
\|M\|_{L^{p}(\mathcal{X})}^{p} \leq \sum_{k=0}^{\infty} \int_{R_{k}\left(B_{\rho}\right)}|M(x)|^{p} d \mu(x) \lesssim \sum_{k=0}^{\infty} 2^{k(1-p)}\left(\eta_{k}\right)^{p} \lesssim 1 .
$$

This verifies (ii).

To obtain (iii), for any $f \in \operatorname{Lip}(1 / p-1)$, we first verify $M\left(f-f_{B_{\rho}}\right) \in L^{1}(\mathcal{X})$. In fact, for all $k \in \mathbb{N} \cup\{0\}$, by Remark 2.1 (b), (1.4) and $f \in \operatorname{Lip}(1 / p-1)$, we have

$$
\begin{aligned}
& \sup _{x \in R_{k}\left(B_{\rho}\right)}\left|f(x)-f_{B_{\rho}}\right| \\
& \quad \lesssim \sup _{x \in B_{\rho}\left(x_{0}, 2^{k} r\right)}\left|f(x)-f\left(x_{0}\right)\right| \lesssim\|f\|_{\operatorname{Lip}(1 / p-1)} 2^{k(1 / p-1)}\left[\mu\left(B_{\rho}\right)\right]^{1 / p-1},
\end{aligned}
$$

from which together with (2.8) and (1.7), we deduce that

$$
\begin{aligned}
& \int_{\mathcal{X}}|M(x)|\left|f(x)-f_{B_{\rho}}\right| d \mu(x) \\
& \quad \leq \sum_{k=0}^{\infty} \sup _{x \in R_{k}\left(B_{\rho}\right)}\left|f(x)-f_{B_{\rho}}\right| \int_{R_{k}\left(B_{\rho}\right)}|M(x)| d \mu(x) \\
& \quad \lesssim\|f\|_{\operatorname{Lip}(1 / p-1)} \sum_{k=0}^{\infty} 2^{k(1 / p-1)} \eta_{k} \lesssim\|f\|_{\operatorname{Lip}(1 / p-1)} .
\end{aligned}
$$

This together with $M \in L^{1}(\mathcal{X})$ gives that $M f \in L^{1}(\mathcal{X})$. Thus, for any $f \in$ $\operatorname{Lip}(1 / p-1)$, if we define $\langle M, f\rangle=\int_{\mathcal{X}} M(x) f(x) d \mu(x)$, then by $\int_{\mathcal{X}} M(x) d \mu(x)=$ 
0, we have $\langle M, f\rangle=\int_{\mathcal{X}} M(x)\left[f(x)-f_{B_{\rho}}\right] d \mu(x)$, which together with (2.9) indicates that $|\langle M, f\rangle| \lesssim\|f\|_{\operatorname{Lip}(1 / p-1)}$. In this sense, we have $M \in(\operatorname{Lip}(1 / p-1))^{*}$ and $\|M\|_{(\operatorname{Lip}(1 / p-1))^{*}} \lesssim 1$, which completes the proof of Proposition 2.2.

To verify Theorem 2.2, we also need the following result.

Lemma 2.2. Let $0<p<1 \leq q \leq \infty$. Then there exists a constant $C>0$ such that for all $f \in L^{q}(\mathcal{X})$ with bounded support and $\int_{\mathcal{X}} f(x) d \mu(x)=0$, $f \in(\operatorname{Lip}(1 / p-1))^{*}$ and $\|f\|_{(\operatorname{Lip}(1 / p-1))^{*}} \leq C\|f\|_{H^{p}(\mathcal{X})}$.

Proof. Let $f \in L^{q}(\mathcal{X})$ be supported in a ball $B_{\rho}$ with $\int_{\mathcal{X}} f(x) d \mu(x)=0$. It is easy to see that $\|f\|_{L^{q}(\mathcal{X})}^{-1}\left[\mu\left(B_{\rho}\right)\right]^{1 / q-1 / p} f$ is a $(p, q)_{\rho}$-atom and thus $f \in$ $(\operatorname{Lip}(1 / p-1))^{*}$ and $f \in H^{p}(\mathcal{X})$ (see $\left[12\right.$, p. 592]). By the definition of $H^{p}(\mathcal{X})$, there exist $\left\{\lambda_{j}\right\}_{j \in \mathbb{N}} \subset \mathbb{C}$ and $(p, q)_{\rho}$-atoms $\left\{a_{j}\right\}_{j \in \mathbb{N}}$ such that $f=\sum_{j=1}^{\infty} \lambda_{j} a_{j}$, which converges in $(\operatorname{Lip}(1 / p-1))^{*}$, and $\left(\sum_{j \in \mathbb{N}}\left|\lambda_{j}\right|^{p}\right)^{1 / p} \lesssim\|f\|_{H^{p}(\mathcal{X})}$. Note that by Remark 2.1 (a), a $(p, q)_{\rho}$-atom is a $(p, q, \eta)_{\rho}$-molecule with $\eta_{k}=0$ for all $k \in \mathbb{N}$. Thus by Proposition 2.2 (iii), we have

$$
\begin{aligned}
\|f\|_{(\operatorname{Lip}(1 / p-1))^{*}} & =\sup _{\|g\|_{\operatorname{Lip}(1 / p-1)} \leq 1}|\langle f, g\rangle| \leq \sup _{\|g\|_{\operatorname{Lip}(1 / p-1)} \leq 1}\left|\sum_{j \in \mathbb{N}} \lambda_{j}\left\langle a_{j}, g\right\rangle\right| \\
& \leq \sup _{\|g\|_{\operatorname{Lip}(1 / p-1)} \leq 1} \sum_{j \in \mathbb{N}}\left|\lambda_{j}\right|\left\|a_{j}\right\|_{(\operatorname{Lip}(1 / p-1))^{*}}\|g\|_{\operatorname{Lip}(1 / p-1)} \\
& \lesssim \sum_{j \in \mathbb{N}}\left|\lambda_{j}\right| \lesssim\|f\|_{H^{p}(\mathcal{X})},
\end{aligned}
$$

which completes the proof of Lemma 2.2.

To end this section, we now present the proof of Theorem 2.2 by invoking some ideas from Coifman and Weiss in [12]; see also [35], [25] and [15].

Proof of Theorem 2.2. Let $M$ be a $(p, q, \eta)_{\rho}$-molecule centered at $B_{\rho}=$ $B_{\rho}\left(x_{0}, r\right)$ for some $x_{0} \in \mathcal{X}$ and $r>0$. Let $\eta_{0}=1$. To decompose $M$ into a

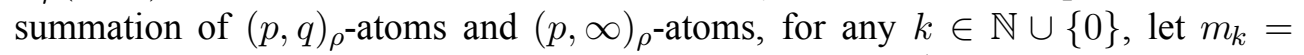
$\int_{R_{k}\left(B_{\rho}\right)} M(x) d \mu(x), \chi_{k}=\chi_{R_{k}\left(B_{\rho}\right)}, \tilde{\chi}_{k}=\left[\mu\left(R_{k}\left(B_{\rho}\right)\right)\right]^{-1} \chi_{k}$ and $M_{k}=M \chi_{k}-$ $m_{k} \widetilde{\chi}_{k}$. Then we have

$$
M=\sum_{k=0}^{\infty} M_{k}+\sum_{k=0}^{\infty} m_{k} \tilde{\chi}_{k} .
$$

Let $N_{j}=\sum_{k=j}^{\infty} m_{k}$. By (2.10) and $N_{0}=\int_{\mathcal{X}} M(x) d \mu(x)=0$, we further obtain

$$
M=\sum_{k=0}^{\infty} M_{k}+\sum_{j=0}^{\infty} N_{j+1}\left(\tilde{\chi}_{j+1}-\tilde{\chi}_{j}\right) .
$$


By (2.8), (M1), (M2) and (1.4), we have that for $k \in \mathbb{N} \cup\{0\}$,

$$
\left\{\int_{\mathcal{X}}\left|M_{k}(x)\right|^{q} d \mu(x)\right\}^{1 / q} \lesssim \eta_{k} 2^{k(1 / p-1)}\left[\mu\left(B_{\rho}\left(x_{0}, 2^{k} r\right)\right)\right]^{1 / q-1 / p} .
$$

This together with the facts that $\operatorname{supp} M_{k} \subset B_{\rho}\left(x_{0}, 2^{k} r\right)$ and $\int_{\mathcal{X}} M_{k}(x) d \mu(x)=0$ indicates that there exists a constant $C>0$, which is independent of $k$ and $M$, such that $\frac{1}{C} 2^{k(1-1 / p)}\left(\eta_{k}\right)^{-1} M_{k}$ for $k \in \mathbb{N} \cup\{0\}$ are $(p, q)_{\rho}$-atoms. By the fact that $\sum_{k=0}^{\infty}\left\|M_{k}\right\|_{L^{1}(\mathcal{X})} \leq 2\|M\|_{L^{1}(\mathcal{X})}$ and Proposition 2.2 (i), we know that the first summation in (2.11) converges in $L^{1}(\mathcal{X})$. If $p<1$, by Lemma 2.2, we have $\left\|M_{k}\right\|_{(\operatorname{Lip}(1 / p-1))^{*}} \lesssim\left\|M_{k}\right\|_{H^{p}(\mathcal{X})}$ for each $k \in \mathbb{N} \cup\{0\}$. From this, we deduce that the first summation in $(2.11)$ converges in $(\operatorname{Lip}(1 / p-1))^{*}$ when $p<1$. On the other hand, by (1.4) and the convention at the end of the introduction, we have

$$
\begin{aligned}
\mu\left(R_{k}\left(\widetilde{B}_{\rho}\right)\right) & =\mu\left(B_{\rho}\left(x_{0}, 2^{k} \mu\left(B_{\rho}\right)\right)\right)-\mu\left(B_{\rho}\left(x_{0}, 2^{k-1} \mu\left(B_{\rho}\right)\right)\right) \\
& \geq\left(C_{5}\right)^{-1} 2^{k} \mu\left(B_{\rho}\right)-C_{5} 2^{k-1} \mu\left(B_{\rho}\right) \geq 2^{k-1} \mu\left(B_{\rho}\right),
\end{aligned}
$$

which together with (1.4) again indicates that

$$
\begin{aligned}
& \left|\widetilde{\chi}_{k+1}(x)-\widetilde{\chi}_{k}(x)\right| \\
& \quad \lesssim\left[2^{k} \mu\left(B_{\rho}\right)\right]^{-1} \lesssim\left[2^{k+1} \mu\left(B_{\rho}\right)\right]^{1 / p-1}\left[\mu\left(B_{\rho}\left(x_{0}, 2^{k+1} r\right)\right)\right]^{-1 / p} .
\end{aligned}
$$

This together with the facts that $\int_{\mathcal{X}}\left[\widetilde{\chi}_{k+1}(x)-\widetilde{\chi}_{k}(x)\right] d \mu(x)=0$ and $\operatorname{supp}\left(\widetilde{\chi}_{k+1}-\right.$ $\left.\tilde{\chi}_{k}\right) \subset B_{\rho}\left(x_{0}, 2^{k+1} r\right)$ implies that there exists a constant $C>0$, which is independent of $k$ and $M$, such that for $k \in \mathbb{N} \cup\{0\}, \frac{1}{C}\left[2^{k+1} \mu\left(B_{\rho}\right)\right]^{1-1 / p}\left(\tilde{\chi}_{k+1}-\tilde{\chi}_{k}\right)$ is a $(p, \infty)_{\rho}$-atom. By (2.8), for $j \in \mathbb{N}$, we have $\left|N_{j}\right| \leq \sum_{k=j}^{\infty} \int_{R_{k}\left(B_{\rho}\right)}\left|M_{k}(x)\right| d \mu(x) \lesssim$ $\sum_{k=j}^{\infty} \eta_{k}\left[\mu\left(B_{\rho}\right)\right]^{1-1 / p}$, which together with (2.12) and an argument similar to the proof for the first summation in (2.11) indicates that the second summation in (2.11) also converges in $L^{1}(\mathcal{X})$ and $(\operatorname{Lip}(1 / p-1))^{*}$ when $p<1$. Further, from (1.6) or (1.7), we deduce that when $p<1$,

$$
\begin{aligned}
& \sum_{k=0}^{\infty} 2^{k(1-p)}\left(\eta_{k}\right)^{p}+\sum_{j=0}^{\infty}\left[N_{j+1}\right]^{p}\left[2^{j} \mu\left(B_{\rho}\right)\right]^{1-p} \\
& \lesssim \sum_{k=0}^{\infty} 2^{k(1-p)}\left(\eta_{k}\right)^{p}+\sum_{j=0}^{\infty} \sum_{k=j+1}^{\infty}\left(\eta_{k}\right)^{p} 2^{j(1-p)} \lesssim 1,
\end{aligned}
$$

and when $p=1, \sum_{k=0}^{\infty} \eta_{k}+\sum_{j=0}^{\infty} N_{j+1} \lesssim 1+\sum_{k=0}^{\infty} k \eta_{k} \lesssim 1$; so $M \in H^{p}(\mathcal{X})$ and $\|M\|_{H^{p}(\mathcal{X})} \lesssim 1$. From this, it is easy to deduce the second conclusion of the theorem, which completes the proof of Theorem 2.2. 


\section{Some General Criteria}

In what follows, let $L_{b}^{\infty}(\mathcal{X})$ be the set of functions in $L^{\infty}(\mathcal{X})$ with bounded support, $\mathcal{C}_{0}(\mathcal{X})$ be the set of continuous functions vanished in infinity for certain fixed $x_{0} \in \mathcal{X}, \mathcal{D}_{0}(\mathcal{X})$ be the set of all functions in $\operatorname{Lip}(\theta)$ with bounded support and $\int_{\mathcal{X}} f(x) d \mu(x)=0$ and $\mathfrak{M}(\mathcal{X})$ be the set of all measurable functions on $\mathcal{X}$. follows.

We first generalize the results of Yabuta [36] to spaces of homogeneous type as

Theorem 3.1. Let $\gamma \in(1, \infty]$. Assume that $T$ is a linear operator from $\mathcal{D}_{0}(\mathcal{X})$ to $\mathfrak{M}(\mathcal{X})$ and satisfies that for any ball $B_{\rho}$ and for every $f \in \mathcal{D}_{0}(\mathcal{X})$, there exist constant $C_{B_{\rho}}>0$, independent of $f$, and constant $C_{B_{\rho}, f}$ such that for certain $s \in(1, \infty)$,

$$
\left[\mu\left(\left\{x \in B_{\rho}:\left|T f(x)-C_{B_{\rho}, f}\right|>\lambda\right\}\right)\right]^{1 / s} \leq C_{B_{\rho}} \lambda^{-1}\|f\|_{L^{\gamma}(\mathcal{X})},
$$

or that

$$
\left\|T f-C_{B_{\rho}, f}\right\|_{L^{1}\left(B_{\rho}\right)} \leq C_{B_{\rho}}\|f\|_{L^{\gamma}(\mathcal{X})} .
$$

(i) If $p \in(1 /(1+\theta), 1], q \in[1, \infty)$ and there exists a constant $C>0$ such that for any $f \in \mathcal{D}_{0}(\mathcal{X})$,

$$
\|T f\|_{L^{q}(\mathcal{X})} \leq C[\operatorname{diam}(\operatorname{supp} f)]^{1 / p}\|f\|_{L^{\infty}(\mathcal{X})},
$$

then $T$ can be extended to a bounded operator from $H^{p}(\mathcal{X})$ to $L^{q}(\mathcal{X})$. Here and in what follows, $\operatorname{diam}(\operatorname{supp} f)=\sup _{x, y \in \operatorname{supp} f} \rho(x, y)$.

(ii) If $p \in(1 /(1+\theta), 1], q \in[p, 1]$ and there exists a constant $C>0$ such that for any $f \in \mathcal{D}_{0}(\mathcal{X})$,

$$
\|T f\|_{H^{q}(\mathcal{X})} \leq C[\operatorname{diam}(\operatorname{supp} f)]^{1 / p}\|f\|_{L^{\infty}(\mathcal{X})},
$$

then $T$ can be extended to a bounded operator from $H^{p}(\mathcal{X})$ to $H^{q}(\mathcal{X})$.

We remark that (3.3) and (3.4) are necessary, which guarantee that $T$ maps all $(p, \infty)_{\rho}$-atoms in $\mathcal{D}_{0}(\mathcal{X})$ boundedly into $L^{q}(\mathcal{X})$ or $H^{q}(\mathcal{X})$, respectively. We also mention that the assumption $q \in[1, \infty]$ in [36, Proposition 1] should be $q \in(1, \infty]$, since $\mathcal{D}_{0}$ is not dense in $L^{1}\left(\mathbb{R}^{n}\right)$.

The following result completes Theorem 3.1, which presents some sufficient conditions for the boundedness of operators from $H^{p}(\mathcal{X})$ to $L^{q}(\mathcal{X})$ with $p \in(1 /(1+$ $\theta), 1)$ and $q \in[p, 1)$. 
Theorem 3.2. Let $p_{0}, q_{0} \in[1, \infty]$ and $T$ be a linear operator bounded from $L^{p_{0}}(\mathcal{X})$ to $L^{q_{0}}(\mathcal{X})$. Let $p \in(1 /(1+\theta), 1)$ and $q \in[p, 1)$. If $T$ satisfies the condition (3.3), then $T$ can be extended to be a bounded operator from $H^{p}(\mathcal{X})$ to $L^{q}(\mathcal{X})$.

The proofs of Theorem 3.1 and Theorem 3.2 are presented in Subsection 3.2 by establishing the pointwise convergence of certain atomic decompositions of $H^{p}(\mathcal{X})$ with $p \in(1 /(1+\theta), 1]$ in [24]; see Proposition 3.1 below.

However, at the end-point case $p=1 /(1+\theta)$, Theorem 3.1 and Theorem 3.2 fail to give the boundedness of $T$ from $H^{1 /(1+\theta)}(\mathcal{X})$ to $L^{q}(\mathcal{X})$ with $q \in[1 /(1+\theta), \infty)$ or $H^{q}(\mathcal{X})$ for $q \in[1 /(1+\theta), 1]$. Instead of this, we have the following conclusions.

Theorem 3.3. Let $p_{0} \in[1, \infty), q_{0} \in[1, \infty)$ and $T$ be a linear operator bounded from $L^{p_{0}}(\mathcal{X})$ to $L^{q_{0}}(\mathcal{X})$.

(i) If $p \in[1 /(1+\theta), 1], q \in[1, \infty)$ and there exists a constant $C>0$ such that for any $(p, \infty) \rho^{- \text {atom }} a,\|T a\|_{L^{q}(\mathcal{X})} \leq C$, then $T$ is bounded from $H^{p}(\mathcal{X})$ to $L^{q}(\mathcal{X})$

(ii) If $p \in[1 /(1+\theta), 1], q \in[p, 1]$ and there exists a constant $C>0$ such that for any $(p, \infty) \rho_{\rho^{-a t o m}} a,\|T a\|_{H^{q}(\mathcal{X})} \leq C$, then $T$ is bounded from $H^{p}(\mathcal{X})$ to $H^{q}(\mathcal{X})$.

(iii) If $p \in[1 /(1+\theta), 1), q \in[p, 1)$ and there exist a constants $C>0$ and $\eta$ satisfying $\sum_{j \in \mathbb{N}} 2^{j(1-q)}\left(\eta_{j}\right)^{q}<\infty$ such that for any $(p, \infty) \rho^{- \text {atom } a, \frac{1}{C} T a}$

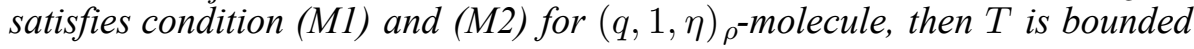
from $H^{p}(\mathcal{X})$ to $L^{q}(\mathcal{X})$.

The proof of Theorem 3.3 is given in Subsection 3.3. To this end, we establish a "smooth" approximation to the given operator by using Coifman's approximations to the identity, which is stated in Subsection 3.1.

\section{Remark 3.1.}

(a) It is easy to see that the boundedness from $L^{p_{0}}(\mathcal{X})$ to $L^{q_{0}}(\mathcal{X})$ of $T$ implies (3.1) or (3.2). Thus Theorem 3.3 when $p \in(1 /(1+\theta), 1]$ is covered by Theorem 3.1 and Theorem 3.2. Since when $p=1 /(1+\theta)$, the maximal function characterization of $H^{p}(\mathcal{X})$ is not available, it is not so clear that in such a case, how can one still verify the pointwise convergence of the atomic decomposition for $H^{1 /(1+\theta)}(\mathcal{X})$ if $T$ only satisfies (3.1) or (3.2)?

(b) Theorem 3.3 strongly depends on the existence of Coifman's approximation to the identity of order $\theta$ and its uniform boundedness in $L^{q}(\mathcal{X})$ when $q \in[1, \infty]$ and in $H^{q}(\mathcal{X})$ when $q \in[1 /(1+\theta), 1]$; see also Remark 3.3 (b). We also note that when $\mathcal{X}=\mathbb{R}^{m}, \mu$ is the $m$-dimensional Lebesgue measure and $\rho(x, y)=|x-y|^{m}$ for all $x, y \in \mathbb{R}^{m}, H^{m /(m+1)}\left(\mathbb{R}^{m}\right)$ in Theorem 3.3 contains the classical Hardy space $H^{m /(m+1)}\left(\mathbb{R}^{m}\right)$ as proper subspace, and atoms of the latter space have vanishing moments up to order 1; see [32]. 
(c) Theorem 3.2 is new even in $\mathbb{R}^{m}$.

From Theorem 3.3 and Theorem 2.2, we directly deduce the following conclusion, which is a key tool of Section 4.

Corollary 3.1. Let $p_{0} \in[1, \infty), q_{0} \in[1, \infty), p \in[1 /(1+\theta), 1), q \in[p, 1)$ and $T$ be a linear operator bounded from $L^{p_{0}}(\mathcal{X})$ to $L^{q_{0}}(\mathcal{X})$. If there exist a constant $C>0$ and $\eta=\left\{\eta_{j}\right\}_{j \in \mathbb{N}} \subset(0, \infty)$ satisfying $\sum_{j \in \mathbb{N}}\left(\eta_{j}\right)^{q} 2^{j(1-q)}<\infty$ such that for any $(p, \infty)$-atom $a, \frac{1}{C} T a$ is a $(q, 1, \eta)_{\rho}$-molecule, then $T$ is bounded from $H^{p}(\mathcal{X})$ to $H^{q}(\mathcal{X})$ and from $H^{p}(\mathcal{X})$ to $L^{q}(\mathcal{X})$.

\subsection{Approximations to the identity}

It is well-known that if $\rho$ satisfies (1.4) and (1.5), then we can construct the following approximation to the identity of order $\theta$ with bounded support on $(\mathcal{X}, \rho, \mu)$; see [13].

Definition 3.1. A sequence $\left\{S_{k}\right\}_{k \in \mathbb{Z}}$ of linear operators is said to be an approximation to the identity of order $\theta$ with bounded support if there exist $C_{8}, C_{9}>$ 0 such that for all $k \in \mathbb{Z}$ and $x, x^{\prime}, y \in \mathcal{X}, S_{k}(x, y)$, the kernel of $S_{k}$ is a function from $\mathcal{X} \times \mathcal{X}$ into $\mathbb{C}$ satisfying

(S1) $S_{k}(x, y)=0$ if $\rho(x, y)>C_{8} 2^{-k}$ and $\left\|S_{k}\right\|_{L^{\infty}(\mathcal{X} \times \mathcal{X})} \leq C_{9} 2^{k}$;

(S2) $\left|S_{k}(x, y)-S_{k}\left(x^{\prime}, y\right)\right| \leq C_{9} 2^{k(1+\theta)}\left[\rho\left(x, x^{\prime}\right)\right]^{\theta}$;

(S3) $\int_{\mathcal{X}} S_{k}(x, y) d \mu(y)=1$;

(S4) Properties (S2) and (S3) hold with $x$ and $y$ interchanged.

\section{Remark 3.2.}

(a) For convenience sake, without loss of generality, we may assume that $C_{8}, C_{9} \geq$ 1 in what follows. Moreover, in the construction of Coifman's approximations to the identity, there exists a constant $\delta>0$ such that for all $\ell>0$ and $x \in B_{\rho}\left(x_{0}, 2^{-\ell-3} / C_{4}\right), S_{\ell}\left(x, x_{0}\right)>\delta$; see [13].

(b) It is easy to see that for all $k \in \mathbb{Z}, 0<\alpha \leq \theta$ and $x \in \mathcal{X}, S_{k}(x, \cdot) \in \operatorname{Lip}(\alpha)$ and $\left\|S_{k}(x, \cdot)\right\|_{\operatorname{Lip}(\alpha)} \lesssim 2^{k(1+\alpha)}$.

(c) We remark that throughout the whole paper, only in the construction of Coifman's approximations to the identity, Theorem 3.1 and its proof, we need to assume that $\rho$ satisfies (1.5). Since we don't use Theorem 3.1 in this paper any more, then, if a such approximation to the identity is known on $(\mathcal{X}, \rho, \mu)$, we then do not need to assume that $\rho$ satisfies (1.5). 
We now state some facts for such approximations to the identity.

Lemma 3.1. The operator $S_{k}$ is bounded on $L^{p}(\mathcal{X})$ for $p \in[1, \infty]$ uniformly in $k \in \mathbb{N}$; for any $f \in L^{p}(\mathcal{X})$ with $p \in[1, \infty)$, $\left\|S_{k} f-f\right\|_{L^{p}(\mathcal{X})} \rightarrow 0$ as $k \rightarrow \infty$; and for any $f \in \mathcal{D}_{0}(\mathcal{X}),\left\|S_{k} f-f\right\|_{L^{\infty}(\mathcal{X})} \rightarrow 0$ as $k \rightarrow \infty$.

Proof. By (S1), it is easy to see the uniform boundedness of $\left\{S_{k}\right\}_{k \in \mathbb{N}}$ in $L^{p}(\mathcal{X})$ with $p \in[1, \infty]$.

By the Lebesgue differentiation theorem (see $[20$, p. 4]. Here we need to use the fact that $\mu$ is Borel regular) together with a standard argument (see [31, p. 11]), we know that if $f \in L^{p}(\mathcal{X})$ with $p \in[1, \infty)$, then for almost everywhere $x \in \mathcal{X}$, $\lim _{k \rightarrow \infty} S_{k} f(x)=f(x)$. This fact together with $\left|S_{k} f(x)\right| \lesssim \mathcal{M}(f)(x)$, where $\mathcal{M}$ is the Hardy-Littlewood maximal operator, the $L^{p}(\mathcal{X})$-boundedness of $\mathcal{M}$ with $p \in(1, \infty)$ (see [11]) and the Lebesgue dominated convergence theorem gives us that $\lim _{k \rightarrow \infty}\left\|S_{k} f-f\right\|_{L^{p}(\mathcal{X})}=0$ for all $f \in L^{p}(\mathcal{X})$. This conclusion together with the density of bounded functions with bounded support in $L^{1}(\mathcal{X})$ further yields that $\lim _{k \rightarrow \infty}\left\|S_{k} f-f\right\|_{L^{1}(\mathcal{X})}=0$ for all $f \in L^{1}(\mathcal{X})$.

Finally, if $f \in \mathcal{D}_{0}(\mathcal{X})$, noting that $\mathcal{D}_{0}(\mathcal{X}) \subset \operatorname{Lip}(\theta)$, then for all $x \in \mathcal{X}$, we have

$$
\left|S_{k}(f)(x)-f(x)\right|=\left|\int_{\mathcal{X}} S_{k}(x, y)[f(y)-f(x)] d \mu(x)\right| \lesssim\|f\|_{\operatorname{Lip}(\theta)} 2^{-k \theta} \rightarrow 0,
$$

as $k \rightarrow \infty$, which completes the proof of Lemma 3.1.

For $p \leq 1$, we have the following conclusion.

Lemma 3.2. Let $1 /(1+\theta) \leq p \leq 1 \leq q \leq \infty, p<q$ and $\eta=\left\{\eta_{k}\right\}_{k \in \mathbb{N}} \subset$ $[0, \infty)$ satisfying (1.6) or (1.7).

(i) There exists a constant $C>0$ such that for all $k \in \mathbb{N}$ and $(p, \infty) \rho_{\text {-atom a }}$ supported in $B_{\rho}\left(x_{0}, r\right)$ for certain $x_{0} \in \mathcal{X}$ and $r>0, \frac{1}{C} S_{k} a$ is $a(p, \infty)_{\rho^{-}}$ atom supported in $B_{\rho}\left(x_{0}, C_{4} r+C_{4} C_{8} 2^{-k}\right)$.

(ii) Let $M$ be a $(p, q, \eta)$-molecule centered at $B_{\rho}\left(x_{0}, r\right)$ for certain $x_{0} \in \mathcal{X}$ and $r>0$. Then there exists a constant $C>0$ independent of $M$ such that for all $k \in \mathbb{N}$ with $C_{8} 2^{-k} \leq r, \frac{1}{C} S_{k} M$ is a $(p, q, \bar{\eta})_{\rho}$-molecule centered at $B_{\rho}\left(x_{0}, 2 C_{4} r\right)$, where for all $j \in \mathbb{N}, \bar{\eta}_{j}=\sum_{k=j}^{j+2 j_{0}+2} \eta_{k}$ and $j_{0} \in \mathbb{N}$ satisfying $2^{j_{0}-1} \leq C_{4}<2^{j_{0}}$.

(iii) $\left\{S_{k}\right\}_{k \in \mathbb{N}}$ is uniformly bounded from $H^{p}(\mathcal{X})$ to $L^{p}(\mathcal{X})$ and from $H^{p}(\mathcal{X})$ to itself; moreover, for $f \in H^{p}(\mathcal{X}),\left\|S_{k} f-f\right\|_{H^{p}(\mathcal{X})} \rightarrow 0$ as $k \rightarrow \infty$. 


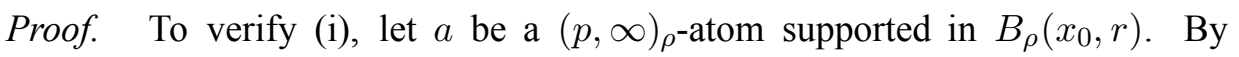
$\int_{\mathcal{X}} a(x) d \mu(x)=0$ and $\int_{\mathcal{X}} S_{k}(x, y) d \mu(x)=1$, it is easy to see that $\int_{\mathcal{X}} S_{k} a(x) d \mu(x)=$ 0 . By (S1) and (1.1) for $\rho$, we have

$$
\operatorname{supp} S_{k} a \subset B_{\rho}\left(x_{0}, C_{4} r+C_{4} C_{8} 2^{-k}\right) \subset B_{\rho}\left(x_{0}, C_{4} r+C_{4} C_{8}\right)=\widetilde{B}_{\rho},
$$

which yields (A1). It remains to verify (A2). If $r \geq 2^{-k}$, by Lemma 3.1 and (1.4), we have

$$
\left|S_{k} a(x)\right| \lesssim\|a\|_{L^{\infty}(\mathcal{X})} \lesssim r^{-1 / p} \lesssim\left[\mu\left(B_{\rho}\left(x_{0}, C_{4} r+C_{4} C_{8} 2^{-k}\right)\right)\right]^{-1 / p}
$$

if $r<2^{-k}$, by $\int_{\mathcal{X}} a(x) d \mu(x)=0$, (S4) and $p \geq 1 /(1+\theta)$, we have

$$
\begin{aligned}
\left|S_{k} a(x)\right| & \lesssim \int_{B_{\rho}\left(x_{0}, r\right)}\left|S_{k}(x, y)-S_{k}\left(x, x_{0}\right)\right||a(y)| d \mu(y) \\
& \lesssim 2^{k(1+\theta)} r^{\theta+1-1 / p} \lesssim\left[\mu\left(B_{\rho}\left(x_{0}, C_{4} r+C_{4} C_{8} 2^{-k}\right)\right)\right]^{-1 / p}
\end{aligned}
$$

Thus, there exists a constant $C>0$ independent of $k$ such that $\frac{1}{C} S_{k} a$ is a $(p, \infty)_{\rho^{-}}$ atom.

To prove (ii), let $k \in \mathbb{N}$ satisfy $C_{8} 2^{-k}<r$ and $M$ be a $(p, q, \eta)_{\rho}$-molecule centered at $B_{\rho}=B_{\rho}\left(x_{0}, r\right)$ for some $x_{0} \in \mathcal{X}$ and $r>0$. By Lemma 3.1, it is easy to see that $S_{k} M \in L^{q}(\mathcal{X})$ and

$$
\left\|S_{k} M\right\|_{L^{q}(\mathcal{X})} \lesssim\|M\|_{L^{q}(\mathcal{X})} \lesssim\left[\mu\left(B_{\rho}\right)\right]^{1 / q-1 / p} \lesssim\left[\mu\left(B_{\rho}\left(x_{0}, 2 C_{4} r\right)\right)\right]^{1 / q-1 / p}
$$

which yields (M1). By Proposition 2.2 (i), Lemma 3.1 and $\int_{\mathcal{X}} S_{k}(x, y) d \mu(x)=1$, we have $\int_{\mathcal{X}} S_{k} M(x) d \mu(x)=0$, which gives (M3). It remains to verify (M2). For all $j \in \mathbb{N}$, by the Hölder inequality, (S1) and (1.4), we have

$$
\begin{aligned}
& \left\{\int_{R_{j}\left(B_{\rho}\left(x_{0}, 2 C_{4} r\right)\right)}\left|S_{k} M(x)\right|^{q} d \mu(x)\right\}^{1 / q} \\
& \quad \lesssim 2^{k / q}\left\{\int_{R_{j}\left(B_{\rho}\left(x_{0}, 2 C_{4} r\right)\right)} \int_{B_{\rho}\left(x, C_{8} 2^{-k}\right)}|M(y)|^{q} d \mu(y) d \mu(x)\right\}^{1 / q} .
\end{aligned}
$$

Let $j_{0} \in \mathbb{N}$ satisfy $2^{j_{0}-1} \leq C_{4}<2^{j_{0}}$. For all $x \in R_{j}\left(B_{\rho}\left(x_{0}, 2 C_{4} r\right)\right)$ and $y \in$ $B_{\rho}\left(x, C_{8} 2^{-k}\right)$, by (1.1) for $\rho, C_{8} 2^{-k}<r$ and (1.4), we have $2^{j-1} r \leq \rho\left(x_{0}, y\right) \leq$ $2^{j+2 j_{0}+2} r$, which together with the Minkowski inequality, (1.4) and (M2) gives that 


$$
\begin{aligned}
& \left\{\int_{R_{j}\left(B_{\rho}\left(x_{0}, 2 C_{4} r\right)\right)}\left|S_{k} M(x)\right|^{q} d \mu(x)\right\}^{1 / q} \\
& \quad \lesssim\left\{\int_{2^{j-1} r \leq \rho\left(x_{0}, y\right)<2^{j+2 j_{0}+2} r}|M(y)|^{q} d \mu(y)\right\}^{1 / q} \\
& \quad \lesssim \sum_{k=j}^{j+2 j_{0}+2}\left\{\int_{2^{k-1} r \leq \rho\left(x_{0}, y\right)<2^{k} r}|M(y)|^{q} d \mu(y)\right\}^{1 / q} \\
& \quad \lesssim\left[\mu\left(B_{\rho}\left(x_{0}, 2 C_{4} r\right)\right)\right]^{1 / q-1 / p_{2}} 2^{j(1 / q-1)} \sum_{k=j}^{j+2 j_{0}+2} \eta_{k} .
\end{aligned}
$$

Let $\bar{\eta}_{j}=\sum_{k=j}^{j+2 j_{0}+2} \eta_{k}$ for all $j \in \mathbb{N}$. Then by (1.6), $\sum_{j=1}^{\infty} j \bar{\eta}_{j}=\sum_{j=1}^{\infty} j \sum_{k=j}^{j+2 j_{0}+2}$ $\eta_{k} \lesssim \sum_{k=1}^{\infty} k \eta_{k} \lesssim 1$, or when $p<1$, by (1.7),

$$
\sum_{j=1}^{\infty}\left(\bar{\eta}_{j}\right)^{p} 2^{j(1-p)} \leq \sum_{j=1}^{\infty} 2^{j(1-p)} \sum_{k=j}^{j+2 j_{0}+2}\left(\eta_{k}\right)^{p} \lesssim \sum_{k=1}^{\infty}\left(\eta_{k}\right)^{p} 2^{k(1-p)} \lesssim 1,
$$

which indicates that $\bar{\eta}=\left\{\bar{\eta}_{j}\right\}_{j \in \mathbb{N}}$ satisfies (1.6) or when $p<1$, (1.7). This finishes the proof of (ii).

To verify (iii), let $f \in L_{b}^{\infty}(\mathcal{X})$ with $\int_{\mathcal{X}} f(x) d \mu(x)=0$. By the definition of $H^{p}(\mathcal{X})$, there exist $(p, \infty)_{\rho}$-atoms $\left\{a_{j}\right\}_{j \in \mathbb{N}}$ and $\left\{\lambda_{j}\right\}_{j \in \mathbb{N}} \subset \mathbb{C}$ such that $f=$ $\sum_{j \in \mathbb{N}} \lambda_{j} a_{j}$, which converges in $L^{1}(\mathcal{X})$ when $p=1$ or in $(\operatorname{Lip}(1 / p-1))^{*}$ when $p<1$, and $\sum_{j \in \mathbb{N}}\left|\lambda_{j}\right|^{p} \lesssim\|f\|_{H^{p}(\mathcal{X})}^{p}$. If $p=1$, by Lemma 3.1, we immediately have

$$
\lim _{N \rightarrow \infty}\left\|S_{k} f-\sum_{j=1}^{N} \lambda_{j}\left(S_{k} a_{j}\right)\right\|_{L^{1}(\mathcal{X})} \lesssim \lim _{N \rightarrow \infty}\left\|f-\sum_{j=1}^{N} \lambda_{j} a_{j}\right\|_{L^{1}(\mathcal{X})}=0,
$$

namely, $S_{k} f(x)=\sum_{j \in \mathbb{N}} \lambda_{j} S_{k} a_{j}(x)$ holds in $L^{1}(\mathcal{X})$. Note that $\frac{1}{C} S_{k} a_{j}$ is a $(1, \infty)_{\rho^{-}}$ atom. Thus, $\left\|S_{k} f\right\|_{H^{1}(\mathcal{X})} \lesssim \sum_{j \in \mathbb{N}}\left|\lambda_{j}\right| \lesssim\|f\|_{H^{1}(\mathcal{X})}$, which together with $H^{1}(\mathcal{X}) \subset$ $L^{1}(\mathcal{X})$ further implies $S_{k}$ is uniformly bounded from $H^{1}(\mathcal{X})$ to $L^{1}(\mathcal{X})$ in $k \in \mathbb{N}$.

When $1 /(1+\theta) \leq p<1$, from (S1) and Remark 3.2 (b), it follows that for all $x \in \mathcal{X}, S_{k} f(x)=\left\langle f, S_{k}(x, \cdot)\right\rangle=\lim _{N \rightarrow \infty}\left\langle\sum_{j=1}^{N} \lambda_{j} a_{j}, S_{k}(x, \cdot)\right\rangle=\sum_{j \in \mathbb{N}} \lambda_{j} S_{k} a_{j}(x)$, where $\langle\cdot, \cdot\rangle$ denotes the dual pair between $(\operatorname{Lip}(1 / p-1))^{*}$ and $\operatorname{Lip}(1 / p-1)$, which

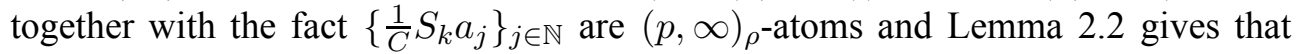
$S_{k} f \in H^{p}(\mathcal{X})$ and $\left\|S_{k} f\right\|_{H^{p}(\mathcal{X})} \lesssim\left\{\sum_{j \in \mathbb{N}}\left|\lambda_{j}\right|^{p}\right\}^{1 / p} \lesssim\|f\|_{H^{p}(\mathcal{X})}$. Since $S_{k} f$ is a function, we then have $S_{k} f \in L^{p}(\mathcal{X})$ and $\left\|S_{k} f\right\|_{L^{p}(\mathcal{X})} \lesssim\|f\|_{H^{p}(\mathcal{X})}$. 
From Lemma 3.1 and (3.5), it follows that if $a$ is a $(p, \infty) \rho^{\text {-atom, then }}$

$$
\left\|S_{k} a-a\right\|_{L^{2}(\mathcal{X})}^{-1}\left[\mu\left(\widetilde{B}_{\rho}\right)\right]^{1 / 2-1 / p}\left(S_{k} a-a\right)
$$

is a $(p, 2) \rho$-atom, which together with Lemma 3.1 again gives that

$$
\left\|S_{k} a-a\right\|_{H^{p}(\mathcal{X})} \lesssim\left[\mu\left(\widetilde{B}_{\rho}\right)\right]^{1 / p-1 / 2}\left\|S_{k} a-a\right\|_{L^{2}(\mathcal{X})} \rightarrow 0, \quad k \rightarrow \infty .
$$

On the other hand, for $k \in \mathbb{N}$ and any $N \in \mathbb{N}$, we have

$$
\left\|S_{k} f-f\right\|_{H^{p}(\mathcal{X})}^{p} \leq \sum_{j=1}^{N}\left|\lambda_{j}\right|^{p}\left\|S_{k} a_{j}-a_{j}\right\|_{H^{p}(\mathcal{X})}^{p}+\sum_{j=N+1}^{\infty}\left|\lambda_{j}\right|^{p} .
$$

By (3.6), we then easily obtain that $\lim _{k \rightarrow \infty}\left\|S_{k} f-f\right\|_{H^{p}(\mathcal{X})}=0$. A density argument then completes the proof of Lemma 3.2.

Moreover, we have the following density results.

Lemma 3.3. If $p \in[1 /(1+\theta), 1]$, then $\mathcal{D}_{0}(\mathcal{X})$ is a density subset of $H^{p}(\mathcal{X})$; if $p \in(1, \infty)$, then $\mathcal{D}_{0}(\mathcal{X})$ is a density subset of $L^{p}(\mathcal{X})$; and if $p=\infty$, then $\mathcal{D}_{0}(\mathcal{X})$ is a density subset of $\mathcal{C}_{0}(\mathcal{X})$.

Proof. Let $f \in L_{b}^{\infty}(\mathcal{X})$ with $\int_{\mathcal{X}} f(x) d \mu(x)=0$ and $\operatorname{supp} f \in B_{\rho}\left(x_{0}, r\right)$ for certain $x \in \mathcal{X}$ and $r \in(0, \infty)$. Then $S_{k} f \in \mathcal{D}_{0}(\mathcal{X})$. In fact, by (S1), $\operatorname{supp} S_{k} f \subset$ $B_{\rho}\left(x_{0}, C_{3} r+C_{8} C_{3}\right)$; by (S3), $\int_{\mathcal{X}} S_{k} f(x) d \mu(x)=\int_{\mathcal{X}} f(x) d \mu(x)=0$; and by (S2), for each $k \in \mathbb{N}$ and all $x, y \in \mathcal{X}$,

$$
\begin{aligned}
& \left|S_{k} f(x)-S_{k} f(y)\right| \\
& \quad \leq \int_{B_{\rho}\left(x_{0}, r\right)}\left|S_{k}(x, z)-S_{k}(y, z)\left\|f(z) \mid d \mu(z) \lesssim 2^{k(1+\theta)}[\rho(x, y)]^{\theta}\right\| f \|_{L^{1}(\mathcal{X})} .\right.
\end{aligned}
$$

If $p \in(1, \infty)$, since the set of all functions $f \in L_{b}^{\infty}(\mathcal{X})$ with $\int_{\mathcal{X}} f(x) d \mu(x)=0$ is a density subset of $L^{p}(\mathcal{X})$, then by Lemma $3.1, \mathcal{D}_{0}(\mathcal{X})$ is dense in $L^{p}(\mathcal{X})$. If $p=\infty$, since the set of all continuous function with bounded support and $\int_{\mathcal{X}} f(x) d \mu(x)=0$ is dense in $\mathcal{C}_{0}(\mathcal{X})$, by Lemma $3.1, \mathcal{D}_{0}(\mathcal{X})$ is dense in $\mathcal{C}_{0}(\mathcal{X})$. If $p \in[1 /(1+\theta), 1]$, from Definition 2.4 , it follows that $L_{b}^{\infty}(\mathcal{X})$ is a density subset of $H^{p}(\mathcal{X})$ for $p \in[1 /(1+\theta), 1]$, which together with Lemma 3.2 (iii) indicates that $\mathcal{D}_{0}(\mathcal{X})$ is dense in $H^{p}(\mathcal{X})$. This completes the proof of Lemma 3.1.

\subsection{Proofs of Theorem 3.1 and Theorem 3.2}

To prove Theorem 3.1 and Theorem 3.2, we need the following conclusion. 
Proposition 3.1. Let $p \in(1 /(1+\theta), 1]$. Then there exists a constant $C>0$ such that for any $f \in \mathcal{D}_{0}(\mathcal{X})$, there exist $\left\{\lambda_{j}\right\}_{j \in \mathbb{N}} \subset \mathbb{C}$ and $(p, \infty)_{\rho}$-atoms $\left\{a_{j}\right\}_{j \in \mathbb{N}} \subset$ $\mathcal{D}_{0}(\mathcal{X})$ satisfying that $\left\{\sum_{j \in \mathbb{N}}\left|\lambda_{j}\right|^{p}\right\}^{1 / p} \leq C\|f\|_{H^{p}(\mathcal{X})}, \sum_{j \in \mathbb{N}}\left|\lambda_{j}\right|\left|a_{j}\right| \in L^{t}(\mathcal{X})$ for all $t \in[1, \infty]$, and $f=\sum_{j=1}^{\infty} \lambda_{j} a_{j}$ in $(\operatorname{Lip}(1 / p-1))^{*}$ and almost everywhere.

Proof. Throughout the proof of this proposition, we use the notation and definitions same as in the proofs of Lemma 4.2 and Theorem 4.13 in [24].

Let $\gamma \in(0, \theta)$ and $q \in(1 /(1+\gamma), p)$ satisfying $1 /(1+\gamma)<q<p \leq 1$. It is easy to see that for all $f \in \mathcal{D}_{0}(\mathcal{X})$, the $\gamma$-maximal function $f_{\gamma}^{*}$, defined as in (1.11) in [24, p. 273] and denoted simply by $f^{*}$, belongs to $L^{t}(\mathcal{X})$ for all $t \in(1 /(1+\gamma), \infty]$, which can be deduced from its definition when $t \in(1, \infty]$ and from Corollary 2.7 in [24] when $t \in(1 /(1+\gamma), 1]$.

Let $h \in \mathcal{D}_{0}(\mathcal{X})$ with $|h(x)| \leq 1$ for all $x \in \mathcal{X}$. By (4.8) in [24, p. 297], we know that there exist $\left\{\lambda_{i, n}\right\}_{i \in \mathbb{N}, n} \subset \mathbb{C}$ and $(p, \infty)_{\rho}$-atoms $\left\{e_{i, n}\right\}_{i \in \mathbb{N}, n}$ such that $h=\sum_{i=1}^{\infty} \sum_{n} \lambda_{i, n} e_{i, n}$ in the sense of distributions (see [24, p. 297]), and $\sum_{i=1}^{\infty} \sum_{n}\left|\lambda_{i, n}\right|^{p} \lesssim \int_{\mathcal{X}}\left[h^{*}(x)\right]^{q} d \mu(x)$ (see [24, p. 298]). Here and in the rest of the proof of this proposition, $f=g$ in the sense of distributions means that $f$ and $g$ as linear continuous functionals on $E^{\theta}$ are equal, where $E^{\theta}$ is the set of all functions in $\operatorname{Lip}(\beta)$ for certain $0<\beta<\theta$ with bounded support, and is endowed a topology defined as in [24, p. 273] with $\alpha$ therein replaced by $\theta$ here.

From (4.3) in [24, p. 296], (3.38) in [24, p. 293], and (2.19) in [24, p. 278], we deduce that $e_{i, n} \in \mathcal{D}_{0}(\mathcal{X})$; and moreover these estimates together with (2.15) in [24, p. 278] and some estimates in [24, p. 297] tell us that for all $x \in \mathcal{X}$,

$$
\sum_{i=1}^{\infty} \sum_{n}\left|\lambda_{i, n}\right|\left|e_{i, n}(x)\right| \lesssim \sum_{i=1}^{\infty} \epsilon^{i} \chi_{E_{i}}(x) \lesssim 1,
$$

where $E_{i}$ was defined in [24, p.296]. On the other hand, from the fact that with certain constant $c>0, \epsilon^{i q} \mu\left(E_{i}\right) \leq(c+2)^{i} \int_{\mathcal{X}}\left[h^{*}(x)\right]^{q} d \mu(x)$ (see [24, p. 298]), it follows that for all $t \in[1, \infty)$, we have

$$
\begin{aligned}
\left\|\sum_{i=1}^{\infty} \epsilon^{i} \chi_{E_{i}}\right\|_{L^{t}(\mathcal{X})} & \lesssim \sum_{i=1}^{\infty} \epsilon^{i}\left[\mu\left(E_{i}\right)\right]^{1 / t} \lesssim \sum_{i=1}^{\infty} \epsilon^{i(1-q / t)}\left[\epsilon^{i q} \mu\left(E_{i}\right)\right]^{1 / t} \\
& \lesssim\left\{\int_{\mathcal{X}}\left[h^{*}(x)\right]^{q} d \mu(x)\right\}^{1 / t} \sum_{i=1}^{\infty} \epsilon^{i(1-q / t)}(c+2)^{i / t} .
\end{aligned}
$$

Now choosing $\epsilon>0$ as in [24, p. 298], we have $\epsilon^{t-q}(c+2)<1$; thus by (3.7), for $t \in[1, \infty)$,

$$
\left\|\sum_{i=1}^{\infty} \sum_{n}\left|\lambda_{i, n}\right|\left|e_{i, n}\right|\right\|_{L^{t}(\mathcal{X})} \leq\left\{\int_{\mathcal{X}}\left[h^{*}(x)\right]^{q} d \mu(x)\right\}^{1 / t} .
$$


Moreover, by (3.38) in [24, p. 293], and (2.15) and (2.17) in [24, p. 278], for each $x \in \mathcal{X}$ and $i$, there exist only finite $e_{i, n}$ such that $e_{i, n}(x) \neq 0$; thus $\sum_{i=1}^{k} \sum_{n} \lambda_{i, n} e_{i, n}$ is a finite summation. This together with (4.4) in [24, p.296], which says that $\left|H_{k}(x)\right| \lesssim \epsilon^{k}$, and the fact that $h(x)=H_{k}(x)+\sum_{i=1}^{k} \sum_{n} \lambda_{i, n} e_{i, n}(x)$ in [24, p. 298], indicates that

$$
h(x)=\sum_{i=1}^{\infty} \sum_{n} \lambda_{i, n} e_{i, n}(x)
$$

holds for almost everywhere $x \in \mathcal{X}$.

Generally, for any $f \in \mathcal{D}_{0}(\mathcal{X})$, by the proof of Theorem 4.13 in [24], there exists functions $h_{k}$ such that $f=\sum_{k=-\infty}^{\infty} h_{k}$ in the sense of distributions (see $\left[24\right.$, p. 300]). Moreover, for any $m \in \mathbb{N}, f-\sum_{k=-m}^{m} h_{k}=B_{m+1}+G_{-m}$, where $\left|G_{-m}(x)\right| \lesssim 2^{-m}$ for all $x \in \mathcal{X}$ by (3.40) in [24, p. 300], and supp $B_{m} \subset \Omega_{m}=$ $\left\{x \in \mathcal{X}: f^{*}(x)>2^{m}\right\}$ by $(3.38)$ in [24, p. 293] and (2.19) in [24, p. 278]. Since $f^{*} \in L^{\infty}(\mathcal{X})$, then there exists $m_{0} \in \mathbb{N}$ such that $B_{m}=0$ for all $m>m_{0}$; and thus $h_{m}=B_{m}-B_{m+1}=0$ for all $m>m_{0}$. From this, we deduce that for all $x \in \mathcal{X}$,

$$
f(x)=\sum_{k=-\infty}^{\infty} h_{k}(x)=\sum_{k=-\infty}^{m_{0}} h_{k}(x) .
$$

Since for all $k \in \mathbb{Z}$ and $k \leq m_{0}, h_{k}=B_{k}-B_{k+1}$, by (3.38) in [24, p. 293] and (2.19) and (2.15) in [24, p.278], we know $h_{k} \in \mathcal{D}_{0}(\mathcal{X})$. By (4.14) in [24, p. 299], we have $\left|c^{-1} 2^{-k} h_{k}(x)\right| \leq 1$ for all $x \in \mathcal{X}$. Therefore, by (3.7),

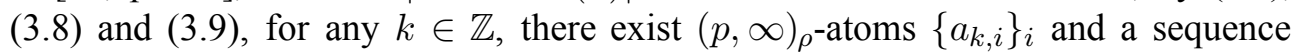
$\left\{\lambda_{k, i}\right\}_{i}$ of numbers such that $c^{-1} 2^{-k} h_{k}=\sum_{i} \lambda_{k, i} a_{k, i}$ in the sense of distributions and almost everywhere; for almost everywhere $x \in \mathcal{X}, \sum_{i}\left|\lambda_{k, i}\right|\left|a_{k, i}(x)\right| \lesssim$ $1 ; \sum_{i}\left|\lambda_{k, i}\right|^{p} \lesssim \int_{\mathcal{X}}\left[\left(c^{-1} 2^{-k} h_{k}\right)^{*}(x)\right]^{q} d \mu(x) \lesssim \mu\left(\Omega_{k}\right) ;$ and for any $t \in[1, \infty)$, $\left\|\sum_{i}\left|\lambda_{k, i}\right|\left|a_{k, i}\right|\right\|_{L^{t}(\mathcal{X})}^{t} \lesssim \int_{\mathcal{X}}\left[\left(c^{-1} 2^{-k} h_{k}\right)^{*}(x)\right]^{q} d \mu(x) \lesssim \mu\left(\Omega_{k}\right)$. Let $\rho_{k, i}=c 2^{k} \lambda_{k, i}$. Then $h_{k}=\sum_{i} \rho_{k, i} a_{k, i}$ in the sense of distributions and almost everywhere; for almost everywhere $x \in \mathcal{X}, \sum_{i}\left|\rho_{k, i}\right|\left|a_{k, i}(x)\right| \lesssim 2^{k} ; \sum_{i}\left|\rho_{k, i}\right|^{p} \lesssim 2^{k p} \mu\left(\Omega_{k}\right)$ (see [24, p. 300]); and for any $t \in[1, \infty),\left\|\sum_{i}\left|\rho_{k, i}\right|\left|a_{k, i}\right|\right\|_{L^{t}(\mathcal{X})}^{t} \lesssim 2^{k t} \mu\left(\Omega_{k}\right)$. Therefore, by (3.10) and Theorem 5.9 in [24], we have $f=\sum_{k=-\infty}^{m_{0}} \sum_{i} \rho_{k, i} a_{k, i}$ in the sense of distributions and almost everywhere, hence in $(\operatorname{Lip}(1 / p-1))^{*}$ and almost everywhere (see the proof of Theorem 5.9 in [24, p. 306]); for almost everywhere $x \in \mathcal{X}$, $\sum_{k=-\infty}^{m_{0}} \sum_{i}\left|\rho_{k, i}\right|\left|a_{k, i}(x)\right| \lesssim 2^{m_{0}}$

$$
\sum_{k=-\infty}^{m_{0}} \sum_{i}\left|\rho_{k, i}\right|^{p} \lesssim \sum_{k} 2^{k p} \mu\left(\Omega_{k}\right) \lesssim \int_{\mathcal{X}}\left[f^{*}(x)\right]^{p} d \mu(x) \lesssim\|f\|_{H^{p}(\mathcal{X})}^{p}
$$

and for any $t \in[1, \infty)$, 


$$
\begin{aligned}
\left\|\sum_{k=-\infty}^{m_{0}} \sum_{i}\left|\rho_{k, i}\right|\left|a_{k, i}\right|\right\|_{L^{t}(\mathcal{X})} & \lesssim \sum_{k=-\infty}^{m_{0}} 2^{k}\left[\mu\left(\Omega_{k}\right)\right]^{1 / t} \\
& \lesssim\left\{\sum_{k=-\infty}^{m_{0}} 2^{k t(1-p) /(t-1)}\right\}^{1-1 / t}\left\{\sum_{k=-\infty}^{m_{0}} 2^{k t p} \mu\left(\Omega_{k}\right)\right\}^{1 / t} \\
& \lesssim\left\{\int_{\mathcal{X}}\left[f^{*}(x)\right]^{t p} d \mu(x)\right\}^{1 / t},
\end{aligned}
$$

which completes the proof of Proposition 3.1.

Proof of Theorem 3.1. To verify (i), let $\widetilde{p}=(1+s) / 2$ and $\psi \in \mathcal{D}_{0}(\mathcal{X})$ with $\operatorname{supp} \psi \subset B_{\rho}=B_{\rho}\left(x_{0}, r\right)$ for certain $x_{0} \in \mathcal{X}$ and $r>0$. Then for any $f \in \mathcal{D}_{0}(\mathcal{X})$, by (3.1),

$$
\begin{aligned}
& \left|\int_{\mathcal{X}} \operatorname{Tf}(x) \psi(x) d \mu(x)\right| \\
& \quad=\left|\int_{\mathcal{X}}\left[T f(x)-C_{B_{\rho}, f}\right] \psi(x) d \mu(x)\right| \\
& \quad \leq\left\|T f-C_{B_{\rho}, f}\right\|_{L^{\tilde{p}}\left(B_{\rho}\right)}\|\psi\|_{L^{\tilde{p}^{\prime}(\mathcal{X})}} \lesssim \mu\left(B_{\rho}\right)^{1 / \widetilde{p}-1 / s}\|f\|_{L^{\gamma}(\mathcal{X})}\|\psi\|_{L^{\tilde{p}^{\prime}(\mathcal{X})}} .
\end{aligned}
$$

Since $\mathcal{D}_{0}(\mathcal{X})$ is dense in $L^{\gamma}(\mathcal{X})$ when $\gamma \in(1, \infty)$ or in $\mathcal{C}_{0}(\mathcal{X})$ when $\gamma=\infty$, there exists a function $g_{\psi} \in L^{\gamma^{\prime}}(\mathcal{X})$ when $\gamma \in(1, \infty)$ or a bounded measure $\mu_{\psi}$ when $\gamma=\infty$ such that when $\gamma \in(1, \infty)$, for all $f \in \mathcal{D}_{0}(\mathcal{X}), \int_{\mathcal{X}} T f(x) \psi(x) d \mu(x)=$ $\int_{\mathcal{X}} f(x) g_{\psi}(x) d \mu(x)$, or that when $\gamma=\infty$, for all $f \in \mathcal{D}_{0}(\mathcal{X}), \int_{\mathcal{X}} T f(x) \psi(x) d \mu(x)=$ $\int_{\mathcal{X}} f(x) d \mu_{\psi}(x)$.

On the other hand, for any $f \in \mathcal{D}_{0}(\mathcal{X})$, by Proposition 3.1, there exist a sequence $\left\{\lambda_{j}\right\}_{j \in \mathbb{N}}$ of numbers and $(p, \infty)_{\rho}$-atoms $\left\{a_{j}\right\}_{j \in \mathbb{N}} \subset \mathcal{D}_{0}(\mathcal{X})$ such that $f=\sum_{j=1}^{\infty} \lambda_{j} a_{j}$, which converges almost everywhere and in $(\operatorname{Lip}(1 / p-1))^{*}$, $\sum_{j \in \mathbb{N}}\left|\lambda_{j}\right|^{p} \lesssim\|f\|_{H^{p}(\mathcal{X})}^{p}$ and $\sum_{j=1}^{\infty}\left|\lambda_{j}\right|\left|a_{j}\right| \in L^{\gamma^{\prime}}(\mathcal{X})$.

If $q \in[1, \infty)$, by (3.3), we have $\left\|T a_{j}\right\|_{L^{q}(\mathcal{X})} \lesssim 1$; thus

$$
\left\|\sum _ { j = 1 } ^ { \infty } \left|\lambda _ { j } \left\|T a _ { j } \left|\left\|_{L^{q}(\mathcal{X})} \leq \sum_{j=1}^{\infty}\left|\lambda_{j}\right|\right\| T a_{j}\left\|_{L^{q}(\mathcal{X})} \leq \sum_{j=1}^{\infty}\left|\lambda_{j}\right| \leq\right\| f \|_{H^{p}(\mathcal{X})} .\right.\right.\right.\right.
$$

From this, if $\gamma \in(1, \infty)$, we deduce that

$$
\begin{array}{rl}
\int_{\mathcal{X}} & T f(x) \psi(x) d \mu(x) \\
& =\int_{\mathcal{X}} f(x) g_{\psi}(x) d \mu(x)=\sum_{j=1}^{\infty} \lambda_{j} \int_{\mathcal{X}} a_{j}(x) g_{\psi}(x) d \mu(x) \\
& =\sum_{j=1}^{\infty} \lambda_{j} \int_{\mathcal{X}} T a_{j}(x) \psi(x) d \mu(x)=\int_{\mathcal{X}} \sum_{j=1}^{\infty} \lambda_{j} T a_{j}(x) \psi(x) d \mu(x),
\end{array}
$$


which together with the density of $\mathcal{D}_{0}(\mathcal{X})$ in $L^{q^{\prime}}(\mathcal{X})$ when $q \in(1, \infty)$ or $\mathcal{C}_{0}(\mathcal{X})$ when $q=1$ gives that $T f(x)=\sum_{j=1}^{\infty} \lambda_{j} T a_{j}(x)$ for almost everywhere $x \in \mathcal{X}$, and hence $T f \in L^{q}(\mathcal{X})$ with $\|T f\|_{L^{q}(\mathcal{X})} \lesssim\|f\|_{H^{p}(\mathcal{X})}$. If $\gamma=\infty$, noting that $\sum_{j \in \mathbb{N}}\left|\lambda_{j}\right|\left|a_{j}\right| \in L^{\infty}(\mathcal{X})$ and $\mu_{\psi}$ is a bounded measure, by a similar procedure, we have the same conclusion. This gives (i).

If $q \in[p, 1]$, by (3.4), then $\left\|T a_{j}\right\|_{H^{q}(\mathcal{X})} \lesssim 1$; thus

$$
\left\|\sum_{j=1}^{\infty} \lambda_{j} T a_{j}\right\|_{H^{q}(\mathcal{X})} \leq\left\{\sum_{j=1}^{\infty}\left|\lambda_{j}\right|^{q}\left\|T a_{j}\right\|_{H^{q}(\mathcal{X})}^{q}\right\}^{1 / q} \leq\left\{\sum_{j=1}^{\infty}\left|\lambda_{j}\right|^{q}\right\}^{1 / q} \leq\|f\|_{H^{p}(\mathcal{X})} .
$$

Similarly, for $\psi \in \mathcal{D}_{0}(\mathcal{X}) \subset \operatorname{Lip}(1 / p-1)$, we still have (3.11), which together with the density of $\mathcal{D}_{0}(\mathcal{X})$ in $\operatorname{Lip}(1 / q-1)$ (see [19, Remark 2.30]) gives $T f=$ $\sum_{j=1}^{\infty} \lambda_{j} T a_{j}$ in $H^{q}(\mathcal{X})$. Therefore $T f \in H^{q}(\mathcal{X})$ and $\|T f\|_{H^{q}(\mathcal{X})} \lesssim\|f\|_{H^{p}(\mathcal{X})}$, which completes the proof of Theorem 3.1.

Proof of Theorem 3.2. Let $f \in \mathcal{D}_{0}(\mathcal{X})$. Then by Proposition 3.1, there exist numbers $\left\{\lambda_{j}\right\}_{j \in \mathbb{N}}$ and $(p, \infty) \rho^{\text {-atoms }}\left\{a_{j}\right\}_{j \in \mathbb{N}} \subset \mathcal{D}_{0}(\mathcal{X})$ such that $f=\sum_{j \in \mathbb{N}}^{\infty} \lambda_{j} a_{j}$ in both $(\operatorname{Lip}(1 / p-1))^{*}$ and almost everywhere, $\left\{\sum_{j \in \mathbb{N}}\left|\lambda_{j}\right|^{p}\right\}^{1 / p} \lesssim\|f\|_{H^{p}(\mathcal{X})}$, and $\sum_{j \in \mathbb{N}}\left|\lambda_{j}\right|\left|a_{j}\right| \in L^{p_{0}}(\mathcal{X})$. From this and the boundedness of $T$ from $L^{p_{0}}(\mathcal{X})$ to $L^{q_{0}}(\mathcal{X})$, it is easy to see that $T f=\sum_{j \in \mathbb{N}} \lambda_{j} T a_{j}$ in both $L^{q_{0}}(\mathcal{X})$ and almost everywhere. Thus when $q \in[p, 1)$,

$$
\|T f\|_{L^{q}(\mathcal{X})} \leq\left\{\sum_{j \in \mathbb{N}}\left|\lambda_{j}\right|^{q}\left\|T a_{j}\right\|_{L^{q}(\mathcal{X})}^{q}\right\}^{1 / q} \lesssim\left\{\sum_{j \in \mathbb{N}}\left|\lambda_{j}\right|^{q}\right\}^{1 / q} \lesssim\|f\|_{H^{p}(\mathcal{X})},
$$

which together with a density argument indicates that $T$ can be extended to be a bounded operator from $H^{p}(\mathcal{X})$ to $L^{q}(\mathcal{X})$. This finishes the proof of Theorem 3.2.

\subsection{Proof of Theorem 3.3}

To prove Theorem 3.3, we need to construct a smooth approximation to the given linear operator $T$ by using Coifman's approximations to the identity. To be precise, let $p_{0}, q_{0} \in[1, \infty), T$ be a linear operator bounded from $L^{p_{0}}(\mathcal{X})$ to $L^{q_{0}}(\mathcal{X})$ and $\left\{S_{\ell}\right\}_{\ell \in \mathbb{Z}}$ be the approximation to identity of order $\theta$ with bounded support as in Definition 3.1. For $\ell \in \mathbb{N}$, set

$$
T_{\ell}=S_{\ell} \circ T \circ S_{\ell}
$$

and for all $x, y \in \mathcal{X}$,

$$
K_{\ell}(x, y)=\int_{\mathcal{X}} S_{\ell}(x, u) T\left(S_{\ell}(\cdot, y)\right)(u) d \mu(u) .
$$


Then we have the following conclusions concerning $K_{\ell}, T_{\ell}$ and $T$.

Lemma 3.4. Let $p_{0} \in[1, \infty), q_{0} \in[1, \infty), T$ be a linear operator bounded from $L^{p_{0}}(\mathcal{X})$ to $L^{q_{0}}(\mathcal{X}),\left\{T_{\ell}\right\}_{\ell \in \mathbb{N}}$ be the same as in (3.12) and $\left\{K_{\ell}\right\}_{\ell \in \mathbb{N}}$ be the same as in (3.13).

(i) For all $f \in L^{p_{0}}(\mathcal{X})$ and $x \in \mathcal{X}, T_{\ell} f(x)=\int_{\mathcal{X}} K_{\ell}(x, y) f(y) d \mu(y)$.

(ii) There exists a constant $C>0$ such that for all $\ell \in \mathbb{N},\left\|K_{\ell}\right\|_{L^{\infty}(\mathcal{X} \times \mathcal{X})} \leq$ $C 2^{\ell\left(1 / q_{0}+1 / p_{0}^{\prime}\right)}$.

(iii) There exists a constant $C>0$ such that for all $\ell \in \mathbb{N}, \alpha \in(0, \theta]$ and $x \in \mathcal{X}$, $K_{\ell}(x, \cdot) \in \operatorname{Lip}(\alpha)$ and $\left\|K_{\ell}(x, \cdot)\right\|_{\operatorname{Lip}(\alpha)} \leq C 2^{\ell\left(1 / q_{0}+1 / p_{0}^{\prime}+\alpha\right)}$.

(iv) $\left\{T_{\ell}\right\}_{\ell \in \mathbb{N}}$ is bounded from $L^{p_{0}}(\mathcal{X})$ to $L^{q_{0}}(\mathcal{X})$ with uniformly bound, and moreover, for all $f \in L^{p_{0}}(\mathcal{X}), T f=\lim _{\ell \rightarrow \infty} T_{\ell} f$ holds in $L^{q_{0}}(\mathcal{X})$.

Proof. To prove (i), let $f \in L^{p_{0}}(\mathcal{X})$. By Lemma 3.1, the boundedness of $T$ from $L^{p_{0}}(\mathcal{X})$ to $L^{q_{0}}(\mathcal{X})$ and the Fubini theorem, we have

$$
\begin{aligned}
T_{\ell} f(x) & =\int_{\mathcal{X}} S_{\ell}(x, z) T\left\{\int_{\mathcal{X}} S_{\ell}(\cdot, y) f(y) d \mu(y)\right\}(z) d \mu(z) \\
& =\int_{\mathcal{X}} S_{\ell}(x, z) \int_{\mathcal{X}} T\left(S_{\ell}(\cdot, y)\right)(z) f(y) d \mu(y) d \mu(z)=\int_{\mathcal{X}} K_{\ell}(x, y) f(y) d \mu(y) .
\end{aligned}
$$

To verify (ii), by (3.13), the Hölder inequality and the boundedness from $L^{p_{0}}(\mathcal{X})$ to $L^{q_{0}}(\mathcal{X})$ of $T$, for all $x, y \in \mathcal{X}$, we have

$$
\left|K_{\ell}(x, y)\right| \lesssim\left\|T S_{\ell}(\cdot, y)\right\|_{L^{q_{0}}(\mathcal{X})}\left\|S_{\ell}(x, \cdot)\right\|_{L^{q_{0}^{\prime}}(\mathcal{X})} \lesssim 2^{\ell\left(1 / q_{0}+1 / p_{0}^{\prime}\right)} .
$$

To establish (iii), if $\rho(x, y) \geq 2 C_{8} 2^{-\ell}$, by (ii), we have

$$
\left|K_{\ell}(z, x)-K_{\ell}(z, y)\right| \lesssim 2^{\ell\left(1 / q_{0}+1 / p_{0}^{\prime}\right)} \lesssim 2^{\ell\left(1 / q_{0}+1 / p_{0}^{\prime}+\alpha\right)}[\rho(x, y)]^{\alpha} .
$$

If $\rho(x, y) \leq 2 C_{8} 2^{-\ell}$, then $\operatorname{supp}\left[S_{\ell}(\cdot, x)-S_{\ell}(\cdot, y)\right] \subset B_{\rho}\left(x, 2 C_{4} C_{8} 2^{-\ell}\right)$, which together with (3.13), (S2), the Hölder inequality and the boundedness from $L^{p_{0}}(\mathcal{X})$ to $L^{q_{0}}(\mathcal{X})$ of $T$ indicates that

$$
\begin{aligned}
\left|K_{\ell}(z, x)-K_{\ell}(z, y)\right| & =\left|\int_{\mathcal{X}} T\left[S_{\ell}(\cdot, x)-S_{\ell}(\cdot, y)\right](u) S_{\ell}(z, u) d \mu(u)\right| \\
& \leq\left\|T\left[S_{\ell}(\cdot, x)-S_{\ell}(\cdot, y)\right]\right\|_{L^{q_{0}}(\mathcal{X})}\left\|S_{\ell}(z, \cdot)\right\|_{L^{q_{0}^{\prime}}(\mathcal{X})} \\
& \lesssim 2^{\ell\left(1 / q_{0}+1 / p_{0}^{\prime}+\alpha\right)}[\rho(x, y)]^{\alpha} .
\end{aligned}
$$

To verify (iv), for $f \in L^{p_{0}}(\mathcal{X})$ with bounded support, by Lemma 3.1, we have $\left\|T_{\ell} f\right\|_{L^{q_{0}}(\mathcal{X})}=\left\|S_{\ell} T S_{\ell} f\right\|_{L^{q_{0}}(\mathcal{X})} \lesssim\left\|T S_{\ell} f\right\|_{L^{q_{0}}(\mathcal{X})} \lesssim\left\|S_{\ell} f\right\|_{L^{p_{0}}(\mathcal{X})} \lesssim\|f\|_{L^{p_{0}}(\mathcal{X})}$. 
Moreover, by Lemma 3.1 and the boundedness of $T$ from $L^{p_{0}}(\mathcal{X})$ to $L^{q_{0}}(\mathcal{X})$, we have

$$
\begin{aligned}
\left\|T_{\ell} f-T f\right\|_{L^{q_{0}}(\mathcal{X})} & \leq\left\|S_{\ell} T\left(S_{\ell} f-f\right)\right\|_{L^{q_{0}}(\mathcal{X})}+\left\|S_{\ell} T f-T f\right\|_{L^{q_{0}}(\mathcal{X})} \\
& \lesssim\left\|S_{\ell} f-f\right\|_{L^{p_{0}}(\mathcal{X})}+\left\|S_{\ell} T f-T f\right\|_{L^{q_{0}}(\mathcal{X})},
\end{aligned}
$$

which converges to 0 . This finishes the proof of Lemma 3.4.

Proof of Theorem 3.3. Let $\left\{T_{\ell}\right\}_{\ell \in \mathbb{N}}$ be the same as in (3.12) and $\left\{K_{\ell}\right\}_{\ell \in \mathbb{N}}$ be the same as in (3.13). Then by Lemma 3.4 (iv), $\left\{T_{\ell}\right\}_{\ell \in \mathbb{N}}$ is bounded from $L^{p_{0}}(\mathcal{X})$ to $L^{q_{0}}(\mathcal{X})$ with uniform bound. Let $f \in \mathcal{D}_{0}(\mathcal{X})$ supported in the ball $B\left(x_{0}, r\right)$ for certain $x_{0} \in \mathcal{X}$ and $r \in(0, \infty)$. Then by Lemma 3.4 (iv), we have $T f=\lim _{\ell \rightarrow \infty} T_{\ell} f$ in $L^{q_{0}}(\mathcal{X})$, which together with the Riesz lemma implies that there exists a subsequence $\left\{\ell_{j}\right\}_{j \in \mathbb{N}}$, where we may assume that $\ell_{j}=j$ for all $j \in \mathbb{N}$ without loss of generality, such that for almost $x \in \mathcal{X}$,

$$
T f(x)=\lim _{\ell \rightarrow \infty} T_{\ell} f(x) .
$$

Moreover, we have $f \in H^{p}(\mathcal{X})$, and hence there exist $\left\{\lambda_{j}\right\}_{j \in \mathbb{N}} \subset \mathbb{C}$ and $(p, \infty)_{\rho^{-}}$ atoms $\left\{a_{j}\right\}_{j \in \mathbb{N}}$ such that $f=\sum_{j \in \mathbb{N}} \lambda_{j} a_{j}$, which converges in $H^{p}(\mathcal{X})$, and

$$
\sum_{j \in \mathbb{N}}\left|\lambda_{j}\right|^{p} \leq 2\|f\|_{H^{p}(\mathcal{X})}^{p}
$$

If $q \geq 1$, to verify (i), by Lemma 3.1 and Lemma 3.2 (i), it is easy to see that $\left\|T_{\ell} a_{j}\right\|_{L^{q}(\mathcal{X})} \lesssim\left\|T S_{\ell} a_{j}\right\|_{L^{q}(\mathcal{X})} \lesssim 1$, which together with $p \leq 1$ and (3.15) indicates that $\sum_{j \in \mathbb{N}} \lambda_{j} T_{\ell} a_{j} \in L^{q}(\mathcal{X})$ and

$$
\left\|\sum_{j \in \mathbb{N}} \lambda_{j} T_{\ell} a_{j}\right\|_{L^{q}(\mathcal{X})} \leq \sum_{j \in \mathbb{N}} \mid \lambda_{j}\left\|T_{\ell} a_{j}\right\|_{L^{q}(\mathcal{X})} \leq\left\{\sum_{j \in \mathbb{N}}\left|\lambda_{j}\right|^{p}\right\}^{1 / p} \lesssim\|f\|_{H^{p}(\mathcal{X})} .
$$

By Lemma 3.4 (iii), we have $K_{\ell}(x, \cdot) \in \operatorname{Lip}(1 / p-1)$ which together with Lemma 3.4 (i) indicates that for any $x \in \mathcal{X}$,

$$
\begin{aligned}
T_{\ell} f(x) & =\int_{\mathcal{X}} K_{\ell}(x, y) f(y) d \mu(y)=\left\langle K_{\ell}(x, \cdot), \sum_{j \in \mathbb{N}} \lambda_{j} a_{j}\right\rangle \\
& =\sum_{j \in \mathbb{N}} \lambda_{j}\left\langle K_{\ell}(x, \cdot), a_{j}\right\rangle=\sum_{j \in \mathbb{N}} \lambda_{j} T_{\ell} a_{j}(x) .
\end{aligned}
$$

From this we deduce that $T_{\ell} f \in L^{q}(\mathcal{X})$ and $\left\|T_{\ell} f\right\|_{L^{q}(\mathcal{X})} \lesssim\|f\|_{H^{p}(\mathcal{X})}$, which together with (3.14) gives that $\|T f\|_{L^{q}(\mathcal{X})} \lesssim \liminf _{\ell \rightarrow \infty}\left\|T_{\ell} f\right\|_{L^{q}(\mathcal{X})} \lesssim\|f\|_{H^{p}(\mathcal{X})}$. This via a density argument gives (i). 
If $q \leq 1$, to verify (ii), by Lemma 3.2 (i) and (iii), we have $\left\|T_{\ell} a\right\|_{H^{q}(\mathcal{X})} \lesssim$ $\left\|T S_{\ell} a\right\|_{H^{q}(\mathcal{X})} \lesssim 1$, which together with $p \leq q$ and (3.15) gives that $\sum_{j \in \mathbb{N}} \lambda_{j} T_{\ell} a_{j} \in$ $H^{q}(\mathcal{X})$ and

$\left\|\sum_{j \in \mathbb{N}} \lambda_{j} T_{\ell} a_{j}\right\|_{H^{q}(\mathcal{X})} \lesssim\left\{\sum_{j \in \mathbb{N}}\left|\lambda_{j}\right|^{q}\left\|T_{\ell} a_{j}\right\|_{H^{q}(\mathcal{X})}^{q}\right\}^{1 / q} \lesssim\left\{\sum_{j \in \mathbb{N}}\left|\lambda_{j}\right|^{p}\right\}^{1 / p} \lesssim\|f\|_{H^{p}(\mathcal{X})}$.

A similar argument tells us that (3.16) still holds for all $x \in \mathcal{X}$ and thus $T_{\ell} f \in$ $H^{q}(\mathcal{X})$ and $\left\|T_{\ell} f\right\|_{H^{q}(\mathcal{X})} \lesssim\|f\|_{H^{p}(\mathcal{X})}$ for all $\ell \in \mathbb{N}$. For $\ell \in \mathbb{N}$, let $\nu_{\ell}=\| S_{\ell} f-$ $f \|_{L^{\infty}(\mathcal{X})}^{-1}\left[\mu\left(B_{\rho}\left(x_{0}, C_{4} r+C_{4} C_{8}\right)\right)\right]^{-1 / p}$. Since $\nu_{\ell}\left(S_{\ell} f-f\right)$ is a $(p, \infty) \rho^{\text {-atom, then }}$ by the assumption, Lemma 3.1 and Lemma 3.2 (i), we have

$$
\begin{aligned}
& \left\|T_{\ell} f-T f\right\|_{H^{q}(\mathcal{X})} \\
& \quad \lesssim\left\|T_{\ell} f-S_{\ell} T f\right\|_{H^{q}(\mathcal{X})}+\left\|S_{\ell} T f-T f\right\|_{H^{q}(\mathcal{X})} \\
& \quad \lesssim\left\|T\left(S_{\ell} f-f\right)\right\|_{H^{q}(\mathcal{X})}+\left\|S_{\ell} T f-T f\right\|_{H^{q}(\mathcal{X})} \\
& \quad \lesssim\left[\mu\left(B_{\rho}\left(x_{0}, C_{4} r+C_{4} C_{8}\right)\right)\right]^{-1 / p}\left\|S_{\ell} f-f\right\|_{L^{\infty}(\mathcal{X})}+\left\|S_{\ell} T f-T f\right\|_{H^{q}(\mathcal{X})},
\end{aligned}
$$

which converges to 0 as $\ell$ converges to $\infty$. This indicates that $T f \in H^{q}(\mathcal{X})$ and

$$
\|T f\|_{H^{q}(\mathcal{X})}=\lim _{\ell \rightarrow \infty}\left\|T_{\ell} f\right\|_{H^{q}(\mathcal{X})} \lesssim\|f\|_{H^{p}(\mathcal{X})},
$$

which together with a density argument gives (ii).

If $q<1$, to verify (iii), let $a$ be a $(p, \infty)_{\rho}$-atom. Since $\frac{1}{C} T a$ satisfies (M1) and (M2) for $(q, 1, \eta)_{\rho}$-molecule, assuming $\frac{1}{C} T a$ is centered at the ball $B_{\rho}\left(z_{0}, r_{0}\right)$ for certain $r_{0}>0$ and $z_{0} \in \mathcal{X}$, and letting $M=(2 C)^{-1}(T a-\tilde{\chi})$, where

$$
\tilde{\chi}=\left\{\int_{\mathcal{X}} \operatorname{Ta}(y) d \mu(y)\right\}\left[\mu\left(B_{\rho}\left(z_{0}, r_{0}\right)\right)\right]^{-1} \chi_{B_{\rho}\left(z_{0}, r_{0}\right)},
$$

then $M$ is a $(q, 1, \eta)_{\rho}$-molecule centered at $B_{\rho}\left(z_{0}, r_{0}\right)$. From this and Lemma 3.2 (ii), it follows that there exists a constant $\widetilde{C}>0$ such that $\widetilde{C}^{-1} S_{\ell} M$ is a $(q, 1, \bar{\eta})_{\rho}$-molecule. On the other hand, by Lemma 3.2 (i), we have $\operatorname{supp} S_{\ell} \widetilde{\chi} \subset$ $B_{\rho}\left(z_{0}, C_{4} r_{0}+C_{4} C_{8} 2^{-\ell}\right)$, which together with Lemma 3.1 and (1.4) gives that

$$
\left\|S_{\ell} \tilde{\chi}\right\|_{L^{q}(\mathcal{X})} \lesssim\left\|S_{\ell} \tilde{\chi}\right\|_{L^{\infty}(\mathcal{X})}\left[\mu\left(B_{\rho}\left(z_{0}, C_{4} r_{0}+C_{4} C_{8} 2^{-\ell}\right)\right)\right]^{1 / q} \lesssim 1 .
$$

From this, $S_{\ell} T a=2 C S_{\ell} M+S_{\ell} \widetilde{\chi}$ and Proposition 2.2, we deduce that $S_{\ell} T a \in$ $L^{q}(\mathcal{X})$ and $\left\|S_{\ell} T a\right\|_{L^{q}(\mathcal{X})} \lesssim\left\|S_{\ell} M\right\|_{L^{q}(\mathcal{X})}+\left\|S_{\ell} \tilde{\chi}\right\|_{L^{q}(\mathcal{X})} \lesssim 1$. From this together with $p<q$, (3.15) and Lemma 3.2 (i), it follows that $\sum_{j \in \mathbb{N}} \lambda_{j} T_{\ell} a_{j} \in L^{q}(\mathcal{X})$ and

$$
\left\|\sum_{j \in \mathbb{N}} \lambda_{j} T_{\ell} a_{j}\right\|_{L^{q}(\mathcal{X})}^{q} \lesssim \sum_{j \in \mathbb{N}}\left|\lambda_{j}\right|^{q}\left\|S_{\ell} T\left(S_{\ell} a_{j}\right)\right\|_{L^{q}(\mathcal{X})}^{q} \lesssim \sum_{j \in \mathbb{N}}\left|\lambda_{j}\right|^{q} \lesssim\|f\|_{H^{p}(\mathcal{X})}^{q}
$$


for all $\ell \in \mathbb{N}$. A similar argument indicates (3.16) still holds for all $x \in \mathcal{X}$, and thus $\left\|T_{\ell} f\right\|_{L^{q}(\mathcal{X})} \lesssim\|f\|_{H^{p}(\mathcal{X})}$ for all $\ell \in \mathbb{N}$. This together with (3.14) and the Fatou lemma indicates that $\|T f\|_{L^{q}(\mathcal{X})} \lesssim \liminf _{\ell \rightarrow \infty}\left\|T_{\ell} f\right\|_{L^{q}(\mathcal{X})} \lesssim\|f\|_{H^{p}(\mathcal{X})}$, which together with a density argument gives (iii) and hence finished the proof of Theorem 3.3.

\section{Remark 3.3.}

(a) The basic idea of the proof of Theorem 3.3 comes from Y. Meyer; see [26, Chapter 7] and also [4].

(b) Let $p_{0}, q_{0} \in[1, \infty), p=1 /(1+\theta)$ and $q \in[p, 1)$. If one only assumes that $T$ is bounded from $L^{p_{0}}(\mathcal{X})$ to $L^{q_{0}}(\mathcal{X})$ and maps all $(1 /(1+\theta), \infty) \rho^{-}$ atoms into $L^{q}(\mathcal{X})$ boundedly, then it is still unclear if Theorem 3.3 (iii) is still true? The method used in this paper seems not valid anymore for this case, since $\left\{S_{\ell}\right\}_{\ell \in \mathbb{N}}$ is not bounded on $L^{q}(\mathcal{X})$ when $q \in[p, 1)$. To see this, let $f(x)=\left[\rho\left(x, x_{0}\right)\right]^{-1} \chi_{B_{\rho}\left(x_{0}, 1\right)}(x)$ for $x \neq x_{0}$ and $f\left(x_{0}\right)=0$. By Remark 3.2 (a), we have $S_{\ell} f(x)=\infty$ for $x \in B_{\rho}\left(x_{0},\left(C_{4}\right)^{-2} 2^{-\ell-4}\right)$, and thus $S_{\ell} f \notin L^{q}(\mathcal{X})$.

(c) Theorem 3.2 also holds for $H^{p}\left(\mathbb{R}^{m}\right)$. It is easy to see that the convergence of atomic decompositions for $H^{1}\left(\mathbb{R}^{m}\right)$ in $[9,22]$ coincides with that in Definition 1.2. When $p \in(m /(1+m), 1)$ with $\theta=1 / m$, according to [9, 22], if $f \in H^{p}\left(\mathbb{R}^{m}\right)$, then $f$ has a decomposition $f=\sum_{j \in \mathbb{N}} \lambda_{j} a_{j}$, which converges in the sense of Schwartz distributions, and $\left(\sum_{j \in \mathbb{N}}\left|\lambda_{j}\right|^{p}\right)^{1 / p} \leq 2\|f\|_{H^{p}\left(\mathbb{R}^{m}\right)}$, which indicates that $\sum_{j \in \mathbb{N}} \lambda_{j} a_{j}$ converges to $f$ in the norm of $H^{p}\left(\mathbb{R}^{m}\right)$. From the fact $\left(H^{p}\left(\mathbb{R}^{m}\right)\right)^{*}=\operatorname{Lip}(1 / p-1)$ with equivalent norms, we deduce that $H^{p}\left(\mathbb{R}^{m}\right) \subset(\operatorname{Lip}(1 / p-1))^{*}$ and $\|g\|_{(\operatorname{Lip}(1 / p-1))^{*}} \lesssim\|g\|_{H^{p}\left(\mathbb{R}^{m}\right)}$ for any $g \in$ $H^{p}\left(\mathbb{R}^{m}\right)$, which indicates that $\sum_{j \in \mathbb{N}} \lambda_{j} a_{j}$ converges to $f$ in $(\operatorname{Lip}(1 / p-1))^{*}$; thus in this case, the convergence of atomic decompositions for $H^{p}\left(\mathbb{R}^{m}\right)$ coincides with that in Definition 1.2.

\section{Boundedness of Singular Integral Operators}

Throughout this section, we always assume that $T$ is a linear operator bounded on $L^{q}(\mathcal{X})$ for certain $q \in(1, \infty)$ with kernel $K$, which is locally integrable on $\mathcal{X} \times \mathcal{X} \backslash\{(x, x): x \in \mathcal{X}\}$. Let $T$ also satisfy that for any $f \in L^{q}(\mathcal{X})$ with bounded support and $x \notin \operatorname{supp} f$,

$$
T f(x)=\int_{\mathcal{X}} K(x, y) f(y) d \mu(y) .
$$

Moreover, suppose $T$ satisfies the following properties: 
(K1) $K \in D_{\rho}(\gamma, \eta)$ for certain $\gamma \in[1, \infty]$ and $\eta=\left\{\eta_{k}\right\}_{k \in \mathbb{N}} \subset[0, \infty)$.

(K2) $T^{*} 1=0$, namely, for any $a \in L^{q}(\mathcal{X})$ with bounded support and $\int_{\mathcal{X}} a(x) d \mu(x)$ $=0, \int_{\mathcal{X}} \operatorname{Ta}(x) d \mu(x)=0$.

Recall that if $K$ satisfies the Hörmander condition with respect to $\rho$, namely, there exist constants $C_{K} \geq 2 C_{4}$ and $C>0$ such that for all $x, y \in \mathcal{X}$,

$$
\int_{\rho(x, z)>C_{K} \rho(x, y)}|K(z, x)-K(z, y)| d \mu(z) \leq C,
$$

Coifman and Weiss $[11,12]$ proved that $T$ is bounded from $L^{p}(\mathcal{X})$ with $p \in(1, q]$ to itself, from $L^{1}(\mathcal{X})$ to weak- $L^{1}(\mathcal{X})$ and from $H^{1}(\mathcal{X})$ to $L^{1}(\mathcal{X})$. If $K \in D_{\rho}(\infty, \eta)$ with $\eta_{k} \leq C 2^{-k \epsilon}$ for $k \in \mathbb{N}$, certain $\epsilon \in(0,1]$ and constant $C>0$, and $T^{*} 1=0$, Coifman and Weiss [12] proved that $T$ is bounded on $H^{1}(\mathcal{X})$; and by further assuming that the corresponding truncated singular integrals converge, Macías and Segovia [25] proved that $T$ is bounded on $H^{p}(\mathcal{X})$ for $1 /(1+\epsilon)<p \leq 1$. If $\mathcal{X}=\mathbb{R}^{m}, \rho(x)=|x|^{m}$ for all $x \in \mathbb{R}^{m}, \mu$ is the $m$-dimensional Lebesgue measure, and $K \in D_{\rho}(1, \eta)$ with $\eta_{k}=C 2^{-k \epsilon}$ for $k \in \mathbb{N}$, certain $\epsilon \in(0,1 / m]$ and constant $C>0$, Alverez [2] then proved that every $(p, \infty) \rho^{\text {-atom was mapped by } T \text { into }}$ $L^{p}\left(\mathbb{R}^{m}\right)$ for $1 /(1+\epsilon)<p \leq 1$ or into weak- $L^{p}\left(\mathbb{R}^{m}\right)$ for $p=1 /(1+\epsilon)$ with uniform bounded norms; however, there is a gap in her proof on how to extend $T$ to the whole $H^{p}\left(\mathbb{R}^{m}\right)$, which was pointed out by Bownik [3]. In this section, we seal this gap. Here are the main results of this section.

Theorem 4.1. If $K \in D_{\rho}(\gamma, \eta)$ with $1<\gamma \leq \infty$ and $\eta=\left\{\eta_{k}\right\}_{k \in \mathbb{N}} \subset[0, \infty)$ satisfying (1.6), and $T^{*} 1=0$, then $T$ is bounded on $H^{1}(\mathcal{X})$.

By the definition of the Hardy space $H^{1}(\mathcal{X})$, it is easy to see that $T^{*} 1=0$ is also necessary for $T$ to be bounded on $H^{1}(\mathcal{X})$.

Theorem 4.2. Let $p \in[1 /(1+\theta), 1)$. If $K \in D_{\rho}(1, \eta)$ with $\eta=\left\{\eta_{k}\right\}_{k \in \mathbb{N}} \subset$ $[0, \infty)$ satisfying (1.7). Then $T$ is bounded from $H^{p}(\mathcal{X})$ to $L^{p}(\mathcal{X})$; if further assume that $T^{*} 1=0$, then $T$ is also bounded on $H^{p}(\mathcal{X})$.

Note that if $\eta_{k}=2^{-k \epsilon}$ for $k \in \mathbb{N}$ and certain $\epsilon \in(0, \theta]$, then (1.7) holds if and only if $p>1 /(1+\epsilon)$. Thus, at the endpoint, we have the following conclusion.

Theorem 4.3. If $K \in D_{\rho}(\infty, \eta)$ with $\eta=\left\{\eta_{k}\right\}_{k \in \mathbb{N}} \subset[0, \infty)$ where $\eta_{k} \leq$ $C 2^{-k \epsilon}$ for $k \in \mathbb{N}$, certain $\epsilon \in(0, \theta]$ and constant $C>0$. Then $T$ is bounded from $H^{1 /(1+\epsilon)}(\mathcal{X})$ to weak $-L^{1 /(1+\epsilon)}(\mathcal{X})$.

The proofs of Theorem 4.1 through Theorem 4.3 are presented in Subsection 4.2 below, by using Theorem 3.3, Corollary 3.1, and Theorem 2.2. To prove Theorem 
4.3, we establish a "smooth" approximation to the given singular integral $T$; see Lemma 4.1 below. For applications, in Subsection 4.1, we present some basic properties on the weak regularity in Definition 1.1.

\section{Remark 4.1.}

(a) Let $T^{*}$ be the conjugate operator of $T$. We remark that the boundedness of $T^{*}$ in $B M O(\mathcal{X})$ and in $\operatorname{Lip}(1 / p-1)$ with $p \in(1 /(1+\theta), 1)$ can be easily deduced, respectively, from Theorem 4.1 and Theorem 4.2 , by using the dual relation between $H^{1}(\mathcal{X})$ and $B M O(\mathcal{X})$, and the dual relation between $H^{p}(\mathcal{X})$ and $\operatorname{Lip}(1 / p-1)$ when $p<1$. We omit the details.

(b) Since $\sum_{j \in \mathbb{N}} j \eta_{j}<\infty$ is obvious much weak than $\eta_{j} \lesssim 2^{-j \epsilon}$ for $j \in \mathbb{N}$, Theorem 4.1 is an essential improvement of the corresponding result of Coifman and Weiss [12]. Moreover, since we do not assume the convergence of the truncated integrals, Theorem 4.2 for $p \in(1 /(1+\epsilon), 1)$ also improves the corresponding result of Macías and Segovia [24]. Even if on $\mathbb{R}^{m}$, Theorem 4.2 and 4.3 improve the corresponding results of Alverez [2].

\subsection{Basic properties on the weak regularity}

The class $D_{\rho}(\gamma, \eta)$ in Definition 1.2 is related to the quasi-metric $\rho$. For the quasi-metric $d$, we can also introduce the corresponding class $D_{d}(\gamma, \eta)$. In fact, similarly as in Definition 1.1, we define the class $D_{d}(\gamma, \eta)$ with $C_{4}, R_{j}\left(B_{\rho}\left(x, C_{K} \rho(x\right.\right.$, $y))$ ) and $B_{\rho}\left(x, 2^{j} C_{K} \rho(x, y)\right)$ replaced respectively by $C_{1},\left\{z \in \mathcal{X}: 2^{(j-1) n} C_{K} d(x\right.$, $\left.y) \leq d(x, z)<2^{j n} C_{K} d(x, y)\right\}$ and $B_{d}\left(x, 2^{j n} C_{K} d(x, y)\right)$ in Definition 1.1.

Recall that on $\left(\mathbb{R}^{m}, d, \mu\right)$, if $\eta \in \ell^{1}$ and $0 \leq \eta_{k+1} \leq \eta_{k}$ for all $k \in \mathbb{N}$, the $L^{q}\left(\mathbb{R}^{m}\right)$-boundedness for certain $q \in(1, \infty)$ of singular integrals with kernels $K \in D_{d}(\infty, \eta)$ was included in Chapter 1 in [32], and that on $\left(\mathbb{R}^{m}, d, \mu\right)$ with $d$ being the Euclidean metric and $\mu$ being the Lebesgue measure, the boundedness in $H^{1}\left(\mathbb{R}^{m}\right)$ and $L^{q}\left(\mathbb{R}^{m}\right)$ for certain $q \in(1, \infty)$ of vector-valued singular integrals with kernels $K \in D_{d}(\gamma, \eta)$ and $\eta \in \ell^{1}$ was obtained by Rubio de Francia, Ruiz and Torrea [29].

It is easy to see that if $1 \leq \gamma_{1}<\gamma_{2} \leq \infty$, then $D_{\rho}\left(\gamma_{2}, \eta\right) \subset D_{\rho}\left(\gamma_{1}, \eta\right)$ and $D_{d}\left(\gamma_{2}, \eta\right) \subset D_{d}\left(\gamma_{1}, \eta\right)$. If $K \in D_{\rho}(1, \eta)$, then it is easy to see that $K$ satisfies the Hörmander condition with respect to $\rho$. If $K \in D_{d}(1, \eta)$, then $K$ as well satisfies the Hörmander condition with respect to $d$ which is obtained by replacing $\rho$ and $C_{4}$ in (4.2) with $d$ and $C_{1}$; respectively. Moreover, we remark that if $K$ satisfies the Hörmander condition with respect to $d$, then $K$ also satisfies the Hörmander condition with respect to $\rho$, namely, (4.2). In fact, for all $x, y, z \in \mathcal{X}$ with $\rho(x, z) \geq\left(C_{K}\right)^{n} C_{3}\left(C_{0}\right)^{2} \rho(x, y)$, where $C_{0}$ denotes the constant in Lemma 2.1 , by 
Lemma 2.1, we have

$$
\frac{V(x, y)}{V(x, z)} \leq\left(C_{0}\right)^{2} \frac{\rho(x, y)}{\rho(x, z)} \leq \frac{1}{C_{3}\left(C_{K}\right)^{n}}<1,
$$

which implies that $d(x, y)<d(x, z)$. This together with (1.3) gives

$$
\frac{d(x, y)}{d(x, z)} \leq\left(C_{3}\right)^{1 / n}\left[\frac{V(x, y)}{V(x, z)}\right]^{1 / n} \leq\left[\left(C_{0}\right)^{2} C_{3}\right]^{1 / n}\left[\frac{\rho(x, y)}{\rho(x, z)}\right]^{1 / n} \leq \frac{1}{C_{K}},
$$

which together with (4.2) yields that

$$
\begin{aligned}
& \int_{\rho(x, z)>\left(C_{K}\right)^{n} C_{3}\left(C_{0}\right)^{2} \rho(x, y)}|K(z, x)-K(z, y)| d \mu(z) \\
& \quad \leq \int_{d(x, z)>C_{K} d(x, y)}|K(z, x)-K(z, y)| d \mu(z) \lesssim 1 .
\end{aligned}
$$

Finally, we clarify the relation between $D_{d}(\gamma, \eta)$ and $D_{\rho}(\gamma, \eta)$ as follows.

Proposition 4.1. Let $1 \leq \gamma \leq \infty$ and $\eta=\left\{\eta_{k}\right\}_{k \in \mathbb{N}} \subset[0, \infty)$. Then there exists a constant $C>0$ independent of $\eta$ such that $D_{d}(\gamma, \eta) \subset D_{\rho}\left(\gamma, \eta^{(\gamma)}\right)$, where $\eta^{(\gamma)}=\left\{\eta_{j}^{(\gamma)}\right\}_{j \in \mathbb{N}}$ and $\eta_{j}^{(\gamma)}=C\left\{\sum_{k=j}^{\infty}\left(\eta_{k}\right)^{\gamma}\right\}^{1 / \gamma}$ for $j \in \mathbb{N}$.

Proof. Let $K \in D_{d}(\gamma, \eta)$. For all $j \in \mathbb{N}$ and $x, y \in \mathcal{X}$, let $r_{j}=2^{j} C_{6}\left(C_{7} C_{K}\right)^{n}$ $\rho(x, y)$. Choosing $r>0$ such that $r / 2 \leq \rho(x, y)<r<r_{j} /\left(C_{6}\left(C_{7} C_{K}\right)^{n}\right)$, then by Proposition 2.1 and Corollary 2.1, we have $C_{K} d(x, y)<C_{K} \widetilde{r}<C_{7} C_{K}$ $\left(\frac{C_{6} r}{r_{j}}\right)^{1 / n} \widetilde{r_{j} / C_{6}} \leq \widetilde{r_{j} / C_{6}}$, and thus

$$
\frac{d(x, y)}{\widetilde{r_{j} / C_{6}}}<\left(2 C_{6}\right)^{1 / n} C_{7}\left[\frac{\rho(x, y)}{r_{j}}\right]^{1 / n}=2^{-(j-1) / n}\left(C_{K}\right)^{-1} .
$$

From this, Lemma 2.1 and Remark 2.3, we deduce that

$$
\begin{aligned}
& {\left[\mu\left(B_{\rho}\left(x, 2 r_{j}\right)\right)\right]^{\gamma-1} \int_{r_{j} \leq \rho(x, z)<2 r_{j}}|K(z, x)-K(z, y)|^{\gamma} d \mu(z)} \\
& \quad \lesssim \int_{r_{j} / C_{6} \leq d(x, z)}[V(x, z)]^{\gamma-1}|K(z, x)-K(z, y)|^{\gamma} d \mu(z) \\
& \quad \lesssim \sum_{k=0}^{\infty}\left[\mu\left(B_{d}\left(x, 2^{(k+j) n} C_{K} d(x, y)\right)\right)\right]^{\gamma-1} \\
& \quad \times \int_{2^{(k+j-1) n} C_{K} d(x, y) \leq d(x, z)<2^{(k+j) n} C_{K} d(x, y)}|K(z, x)-K(z, y)|^{\gamma} d \mu(z) \\
& \quad \lesssim \sum_{k=j}^{\infty}\left(\eta_{k}\right)^{\gamma},
\end{aligned}
$$


which indicates $K \in D_{\rho}\left(\gamma, \eta^{(\gamma)}\right)$, and hence completes the proof of Proposition 4.1 .

\subsection{Proofs of Theorem 4.1 through Theorem 4.3}

We begin with the proof of Theorem 4.1.

Proof of Theorem 4.1. Without loss of generality, we may assume that $1<$ $\gamma \leq q$. Let $a$ be a $(1, \infty)_{\rho}$-atom supported in $B_{\rho}=B_{\rho}\left(x_{0}, r\right)$ for some $x_{0} \in \mathcal{X}$ and $r>0$. We now claim that there exists a constant $C>0$ independent of $a$ such that $\frac{1}{C} T a$ is a $(1, \gamma, \widetilde{\eta})_{\rho}$-molecule centered at $B_{\rho}\left(x_{0}, C_{K} r\right)$, where $\widetilde{\eta}_{j}=\sum_{k=j+1}^{\infty} \eta_{k} 2^{j-k}$. If we can prove this, an application of Corollary 3.1 leads to the desired conclusion of the theorem.

In fact, by the $L^{\gamma}(\mathcal{X})$-boundedness of $T$ and (1.4), it is easy to see that

$$
\|T a\|_{L^{\gamma}(\mathcal{X})} \lesssim\|a\|_{L^{\gamma}(\mathcal{X})} \lesssim\left[\mu\left(B_{\rho}\right)\right]^{1 / \gamma-1} \lesssim\left[\mu\left(B_{\rho}\left(x_{0}, C_{K} r\right)\right)\right]^{1 / \gamma-1},
$$

which gives (M1). Since (M3) follows from $T^{*} 1=0$, it remains to verify (M2). For $j \in \mathbb{N}$, by $\int_{\mathcal{X}} a(x) d \mu(x)=0$, the Minkowski inequality, $K \in D_{\rho}(\gamma, \eta)$ and (1.4), we obtain

$$
\begin{aligned}
& \left\{\int_{R_{j}\left(B_{\rho}\left(x_{0}, C_{K} r\right)\right)}|T a(x)|^{\gamma} d \mu(x)\right\}^{1 / \gamma} \\
& \leq\left\{\int_{R_{j}\left(B_{\rho}\left(x_{0}, C_{K} r\right)\right)}\left[\int_{B_{\rho}}\left|K(x, y)-K\left(x, x_{0}\right)\right||a(y)| d \mu(y)\right]^{\gamma} d \mu(x)\right\}^{1 / \gamma} \\
& \leq\left[\mu\left(B_{\rho}\right)\right]^{-1} \int_{B_{\rho}}\left\{\int_{R_{j}\left(B_{\rho}\left(x_{0}, C_{K} r\right)\right)}\left|K(x, y)-K\left(x, x_{0}\right)\right|^{\gamma} d \mu(x)\right\}^{1 / \gamma} d \mu(y) \\
& \leq\left[\mu\left(B_{\rho}\right)\right]^{-1} \sum_{k=1}^{\infty} \int_{2^{-k-1} C_{K} r \leq \rho\left(x_{0}, y\right)<2^{-k} C_{K} r} \\
& \quad \times\left\{\int_{2^{j+k-1} C_{K} \rho\left(x_{0}, y\right) \leq \rho\left(x_{0}, x\right)<2^{j+k+1} C_{K} \rho\left(x_{0}, y\right)} d \mu(y)\right. \\
& \left.\left.\quad \times\left|K(x, y)-K\left(x, x_{0}\right)\right|^{\gamma} d \mu(x)\right\}^{1 / \gamma} d \mu(y)\right]_{k=j+1}^{\infty} \eta_{k} 2^{j-k} . \\
& \lesssim\left[\mu\left(B_{\rho}\left(x_{0}, C_{K} r\right)\right)\right]^{1 / \gamma-1} 2^{j(1 / \gamma-1)} \sum_{k=1}^{\infty}
\end{aligned}
$$

By (1.6) for $\eta$, we have

$$
\sum_{j=1}^{\infty} j \widetilde{\eta}_{j}=\sum_{j=1}^{\infty} j \sum_{k=j+1}^{\infty} \eta_{k} 2^{j-k}=\sum_{k=1}^{\infty} \eta_{k} 2^{-k} \sum_{j=1}^{k} j 2^{j} \lesssim \sum_{k=1}^{\infty} k \eta_{k} \lesssim 1,
$$


which yields that $\widetilde{\eta}$ satisfies (1.6), and hence verifies the claim. This finishes the proof of Theorem 4.1.

Proof of Theorem 4.2. Let $a$ be a $(p, \infty) \rho$-atom supported in $B_{\rho}=B_{\rho}\left(x_{0}, r\right)$ for certain $x_{0} \in \mathcal{X}$ and $r>0$, where $1 /(1+\theta) \leq p<1$. We first claim that there exists a constant $C>0$ independent of $a$ such that $\frac{1}{C} T a$ satisfies (M1) and (M2) centered at $B_{\rho}\left(x_{0}, C_{K} r\right)$ with $q=1$ and $\eta$ replaced by $\widetilde{\eta}$, where $\widetilde{\eta}_{j}=\sum_{k=j+1}^{\infty} \eta_{k} 2^{j-k}$ for all $j \in \mathbb{N}$. Note that it is easy to verify that if $\eta$ satisfies (1.7), then $K$ also satisfies the Hörmander condition (4.2). Therefore, by the boundedness of $T$ from $H^{1}(\mathcal{X})$ to $L^{1}(\mathcal{X})$ and (1.4), we have

$$
\|T a\|_{L^{1}(\mathcal{X})} \lesssim\|a\|_{H^{1}(\mathcal{X})} \lesssim\left[\mu\left(B_{\rho}\right)\right]^{1-1 / p} \lesssim\left[\mu\left(B_{\rho}\left(x_{0}, C_{K} r\right)\right)\right]^{1-1 / p},
$$

which gives (M1). For $j \in \mathbb{N}$, by $\int_{\mathcal{X}} a(x) d \mu(x)=0$, the Minkowski inequality, $K \in D_{\rho}(1, \eta)$ and (1.4), we obtain

$$
\begin{aligned}
& \int_{R_{j}\left(B_{\rho}\left(x_{0}, C_{K} r\right)\right)}|\operatorname{Ta}(x)| d \mu(x) \\
& \leq \int_{R_{j}\left(B_{\rho}\left(x_{0}, C_{K} r\right)\right)} \int_{B_{\rho}}\left|K(x, y)-K\left(x, x_{0}\right)\right||a(y)| d \mu(y) d \mu(x) \\
& \leq\left[\mu\left(B_{\rho}\right)\right]^{-1 / p} \sum_{k=1}^{\infty} \int_{2^{-k-1} C_{K} r \leq \rho\left(x_{0}, y\right)<2^{-k} C_{K} r}\left|K(x, y)-K\left(x, x_{0}\right)\right| d \mu(x) d \mu(y) \\
& \quad \times \int_{2^{j+k-1} C_{K} \rho\left(x_{0}, y\right) \leq \rho\left(x_{0}, x\right)<2^{j+k+1} C_{K} \rho\left(x_{0}, y\right)} \\
& \lesssim\left[\mu\left(B_{\rho}\left(x_{0}, C_{K} r\right)\right)\right]^{1-1 / p} \sum_{k=j+1}^{\infty} \eta_{k} 2^{j-k} .
\end{aligned}
$$

By (1.7) for $\eta$, we have

$$
\begin{aligned}
\sum_{j=1}^{\infty} 2^{j(1-p)}\left(\widetilde{\eta}_{j}\right)^{p} & \leq \sum_{j=1}^{\infty} 2^{j} \sum_{k=j+1}^{\infty}\left(\eta_{k}\right)^{p} 2^{-p k} \\
& =\sum_{k=1}^{\infty}\left(\eta_{k}\right)^{p} 2^{-k p} \sum_{j=1}^{k} 2^{j} \leq \sum_{k=1}^{\infty} 2^{k(1-p)}\left(\eta_{k}\right)^{p}<\infty
\end{aligned}
$$

which yields that $\widetilde{\eta}=\left\{\widetilde{\eta}_{j}\right\}_{j \in \mathbb{N}}$ satisfies (1.7), and hence the claim. This together with Theorem 3.3 (iii) completes the proof of Theorem 4.2.

To verify Theorem 4.3, we need the following conclusion. 
Lemma 4.1. Let $q \in(1, \infty), T$ be a linear operator bounded on $L^{q}(\mathcal{X})$ with kernel $K$ as in (4.1) and $\left\{K_{\ell}\right\}_{\ell \in \mathbb{N}}$ be the same as in (3.13). If $K \in D_{\rho}(\infty, \eta)$, where $\eta=\left\{\eta_{k}\right\}_{k \in \mathbb{N}} \subset[0, \infty)$ with $\eta_{k} \leq C 2^{-k \epsilon}$ for $k \in \mathbb{N}$, certain $\epsilon \in(0, \theta]$ and constant $C>0$, then there exists a constant $\widetilde{C}>0$ such that for all $\ell \in \mathbb{N}$, $K_{\ell} \in D_{\rho}(\infty, \widetilde{\eta})$ with $\widetilde{\eta}=\left\{\widetilde{C} 2^{-k \epsilon}\right\}_{k \in \mathbb{N}}$.

Proof. For $K \in D_{\rho}(\infty, \eta)$ with $\eta_{k} \lesssim 2^{-k \epsilon}$, by Definition 1.1 and (1.4), it is easy to see that for all $x, y, z \in \mathcal{X}$ with $\rho(x, z) \geq C_{K} \rho(x, y)$,

$$
|K(z, x)-K(z, y)| \lesssim\left[\frac{\rho(x, y)}{\rho(x, z)}\right]^{\epsilon} \frac{1}{\rho(x, z)} .
$$

On the other hand, to prove Lemma 4.1, it suffices to verify that there exists a constant $\widetilde{C}_{K}>0$ such that (4.3) holds.

We first claim that if $\rho(x, y)>2\left(C_{4}\right)^{2} C_{8} 2^{-\ell}$, for $u \in B_{\rho}\left(x, C_{8} 2^{-\ell}\right)$ and $v \in B_{\rho}\left(y, C_{8} 2^{-\ell}\right)$, by (1.1) for $\rho$, we then have $\rho(x, y) \leq\left(C_{4}\right)^{2}[\rho(x, u)+\rho(u, v)+$ $\rho(v, y)] \leq 2 C_{8}\left(C_{4}\right)^{2} 2^{-\ell}+\left(C_{4}\right)^{2} \rho(u, v)$, which gives that $\rho(u, v)>0$; thus by (S1), we can write

$$
K_{\ell}(x, y)=\int_{\mathcal{X}} \int_{\mathcal{X}} S_{\ell}(x, u) K(u, v) S_{\ell}(v, y) d \mu(u) d \mu(v) .
$$

When $\rho(x, y)>C_{8} 2^{-\ell}$, for $z \in \mathcal{X}$ with $\rho(x, z)>4 C_{K}\left(C_{4}\right)^{3} \rho(x, y)$, which implies that $\rho(z, y)>2\left(C_{4}\right)^{2} C_{8} 2^{-\ell}$, by (4.4), (S1) and $\int_{\mathcal{X}} S_{\ell}(v, x) d \mu(v)=1$, we have

$$
\begin{aligned}
& K_{\ell}(z, x)-K_{\ell}(z, y) \\
& =\int_{B_{\rho}\left(z, C_{8} 2^{-\ell}\right)} \int_{B_{\rho}\left(x, C_{8} 2^{-\ell}\right) \cup B_{\rho}\left(y, C_{8} 2^{-\ell}\right)} S_{\ell}(z, u) \\
& \quad \times[K(u, v)-K(u, x)]\left[S_{\ell}(v, x)-S_{\ell}(v, y)\right] d \mu(v) d \mu(u) .
\end{aligned}
$$

For $u \in B_{\rho}\left(z, C_{8} 2^{-\ell}\right)$ and $v \in B_{\rho}\left(x, C_{8} 2^{-\ell}\right) \cup B_{\rho}\left(y, C_{8} 2^{-\ell}\right)$, by (1.1) for $\rho$, we have $\rho(v, x) \leq 2 C_{4} \rho(x, y), \rho(u, x) \leq C_{4}\left(1+\frac{1}{4 C_{K}\left(C_{4}\right)^{3}}\right) \rho(x, z) \leq 2 C_{4} \rho(x, z)$, and similarly $\rho(x, z) \leq 2 C_{4} \rho(x, u)$, which indicates that $\frac{\rho(x, z)}{2 C_{4}} \leq \rho(x, u) \leq$ $2 C_{4} \rho(x, z)$. This gives that

$$
\frac{\rho(v, x)}{\rho(u, x)} \leq 4\left(C_{4}\right)^{2} \frac{\rho(x, y)}{\rho(x, z)}<1 / C_{K}
$$

which together with (4.3) and (1.4) gives that

$$
\begin{aligned}
& \left|K_{\ell}(z, x)-K_{\ell}(z, y)\right| \\
& \quad \lesssim 2^{2 \ell} \int_{B_{\rho}\left(z, C_{8} 2^{-\ell}\right)} \int_{B_{\rho}\left(x, C_{8} 2^{-\ell}\right) \cup B_{\rho}\left(y, C_{8} 2^{-\ell}\right)}|K(u, v)-K(u, x)| d \mu(v) d \mu(u) \\
& \quad \lesssim\left[\frac{\rho(x, y)}{\rho(x, z)}\right]^{\epsilon} \frac{1}{\rho(x, z)} .
\end{aligned}
$$


When $\rho(x, y) \leq C_{8} 2^{-\ell}$, for $z \in \mathcal{X}$ with $4 C_{K}\left(C_{4}\right)^{3} \rho(x, y)<\rho(x, z) \leq$ $4 C_{K}\left(C_{4}\right)^{3} C_{8} 2^{-\ell}$, by (ii) and (1.4), we have

$$
\left|K_{\ell}(z, x)-K_{\ell}(z, y)\right| \lesssim 2^{\ell(1+\epsilon)}[\rho(x, y)]^{\epsilon} \lesssim\left[\frac{\rho(x, y)}{\rho(x, z)}\right]^{\epsilon} \frac{1}{\rho(x, z)} .
$$

For $z \in \mathcal{X}$ with $\rho(x, z) \geq 4 C_{K}\left(C_{4}\right)^{3} C_{8} 2^{-\ell}$, which implies that $\rho(z, y)>2\left(C_{4}\right)^{2} C_{8}$ $2^{-\ell}$, and therefore (4.5) holds. For $v \in B_{\rho}\left(x, C_{8} 2^{-\ell}\right) \cup B_{\rho}\left(y, C_{8} 2^{-\ell}\right)$ and $u \in$ $B_{\rho}\left(z, C_{8} 2^{-\ell}\right)$, by (1.1) for $\rho$, we have $\rho(v, x) \leq 2 C_{4} C_{8} 2^{-\ell}$, and $\rho(x, z) /\left(2 C_{4}\right) \leq$ $\rho(u, x) \leq 2 C_{4} \rho(x, z)$. Then $\rho(x, v)<\rho(x, u) / C_{K}$. Thus, for $K \in D_{\rho}(\infty, \eta)$, by (4.5), (4.3) and (S2), we have

$$
\begin{aligned}
& \left|K_{\ell}(z, x)-K_{\ell}(z, y)\right| \\
& \quad \lesssim 2^{\ell} 2^{\ell(1+\epsilon)}[\rho(x, y)]^{\epsilon} \int_{B_{\rho}\left(z, C_{8} 2^{-\ell}\right)} \int_{B_{\rho}\left(x, 2 C_{4} C_{8} 2^{-\ell}\right)}|K(u, v)-K(u, x)| d \mu(v) d \mu(u) \\
& \quad \lesssim\left[\frac{\rho(x, y)}{\rho(x, z)}\right]^{\epsilon} \frac{1}{\rho(x, z)}
\end{aligned}
$$

which completes the proof of Lemma 4.1.

Remark 4.2. We remark that the method used in the proof of Lemma 4.1 is the same as in [26] (see also [4]), namely, when $\eta=\left\{\eta_{k}\right\}_{k \in \mathbb{N}}$ with $\eta_{k} \leq C 2^{-k \epsilon}$ for $k \in \mathbb{N}$, certain $\epsilon \in(0, \theta]$ and constant $C>0$, and $K \in D_{\rho}(\infty, \eta)$, we verify that the kernel $K_{\ell}$ of the operator $T_{\ell}=S_{\ell} \circ T \circ S_{\ell}$ for $\ell \in \mathbb{N}$ still belongs to $D_{\rho}(\infty, \widetilde{\eta})$ with $\widetilde{\eta}=\left\{\widetilde{C} 2^{-k \epsilon}\right\}_{k \in \mathbb{N}}$, where $\widetilde{C}>0$ is independent of $\ell$ and $k$. However, it is unclear to us if this is still true for general $\eta$ as in (1.6) or (1.7).

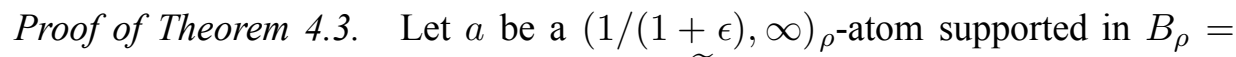
$B_{\rho}(x, r)$ for certain $x \in \mathcal{X}$ and $r>0$. Setting $\widetilde{B}_{\rho}=B_{\rho}\left(x_{0}, 2 C_{K} r\right)$, we have

$\mu(\{x \in \mathcal{X}:|T a(x)|>2 \lambda\}) \leq \mu\left(\left\{x \in \widetilde{B}_{\rho}:|T a(x)|>\lambda\right\}\right)+\mu\left(\left\{x \in \widetilde{B}_{\rho}:|T a(x)|>\lambda\right\}\right)$.

From the Hölder inequality, the boundedness of $T$ from $H^{1}(\mathcal{X})$ to $L^{1}(\mathcal{X})$ and (1.4), it follows that

$$
\int_{\widetilde{B}_{\rho}}|T a(x)|^{1 /(1+\epsilon)} d \mu(x) \lesssim\|T a\|_{L^{1}(\mathcal{X})}^{1 /(1+\epsilon)}\left[\mu\left(B_{\rho}\right)\right]^{\epsilon /(1+\epsilon)} \lesssim\|a\|_{H^{1}(\mathcal{X})}\left[\mu\left(B_{\rho}\right)\right]^{\epsilon /(1+\epsilon)} \lesssim 1,
$$

which leads to that

$$
\lambda^{1 /(1+\epsilon)} \mu\left(\left\{x \in \widetilde{B}_{\rho}:|T a(x)|>\lambda\right\}\right) \leq \int_{\widetilde{B}_{\rho}}|T a(x)|^{1 /(1+\epsilon)} d \mu(x) \lesssim 1 .
$$


For $x \notin \widetilde{B}_{\rho}$, by $K \in D_{\rho}(\infty, \eta),(4.3)$ and (1.4), we have

$$
\begin{aligned}
|T a(x)| & \leq\left[\mu\left(B_{\rho}\right)\right]^{-1 / p} \int_{y \in B_{\rho}}\left|K(x, y)-K\left(x, x_{0}\right)\right| d \mu(y) \\
& \lesssim\left[\mu\left(B_{\rho}\right)\right]^{-(1+\epsilon)} \int_{y \in B_{\rho}}\left[\frac{\rho\left(x_{0}, y\right)}{\rho\left(x_{0}, x\right)}\right]^{\epsilon} \frac{1}{\rho\left(x_{0}, x\right)} d \mu(y) \lesssim\left[\rho\left(x_{0}, x\right)\right]^{-(1+\epsilon)},
\end{aligned}
$$

from which and (1.4), it follows that there exists a constant $C>0$, independent of $a$ and $\lambda$, such that

$\mu\left(\left\{x \notin \widetilde{B}_{\rho}:|T a(x)|>\lambda\right\}\right) \leq \mu\left(\left\{x \in \mathcal{X}:\left[\rho\left(x_{0}, x\right)\right]^{-(1+\epsilon)}>\lambda / C\right\}\right) \lesssim \lambda^{-1 /(1+\epsilon)}$.

Thus, $\mu(\{x \in \mathcal{X}:|T a(x)|>\lambda\}) \lesssim \lambda^{-1 /(1+\epsilon)}$. By Lemma 4.1, this also holds for $T_{\ell}$ uniformly in $\ell \in \mathbb{N}$.

For any $f \in \mathcal{D}_{0}(\mathcal{X})$, there exist a sequence of numbers $\left\{\lambda_{j}\right\}_{j \in \mathbb{N}}$ and $(1 /(1+$ $\theta), \infty)$-atoms $\left\{a_{j}\right\}_{j \in \mathbb{N}}$ such that $f=\sum_{j \in \mathbb{N}} \lambda_{j} a_{j}$ which converges in $H^{p}(\mathcal{X})$ and $\sum_{j \in \mathbb{N}}\left|\lambda_{j}\right|^{p} \leq 2\|f\|_{H^{p}(\mathcal{X})}$. Then by (3.13) and a well-known inequality called addition principle of weak type (see [33]), we have

$$
\begin{aligned}
\lambda^{1 /(1+\epsilon)} \mu\left(\left\{x \in \mathcal{X}:\left|T_{\ell} f(x)\right|>2 \lambda\right\}\right) \\
\quad=\lambda^{1 /(1+\epsilon)} \mu\left(\left\{x \in \mathcal{X}:\left|\sum_{j=1}^{\infty} \lambda_{j} T_{\ell} a(x)\right|>2 \lambda\right\}\right) \\
\quad \lesssim \sum_{j=1}^{\infty}\left|\lambda_{j}\right|^{1 /(1+\epsilon)} \lesssim\|f\|_{H^{1 /(1+\epsilon)}(\mathcal{X})}^{1 /(1+\epsilon)} .
\end{aligned}
$$

On the other hand, by Lemma 3.4 (iii), we have $T f(x)=\lim _{\ell \rightarrow \infty} T_{\ell} f(x)$ in $L^{q}(\mathcal{X})$, and thus, there exists subsequence $\left\{\ell_{j}\right\}_{j \in \mathbb{N}}$ such that $T f(x)=\lim _{j \rightarrow \infty} T_{\ell_{j}} f(x)$ for almost everywhere $x \in \mathcal{X}$. From this and the Fatou lemma, we have for all $\lambda>0$,

$$
\begin{aligned}
& \mu(\{x \in \mathcal{X}:|T f(x)|>\lambda\}) \lesssim \liminf _{j \rightarrow \infty} \mu\left(\left\{x \in \mathcal{X}:\left|T_{\ell_{j}} f(x)\right|>\lambda\right\}\right) \\
& \quad \lesssim\left(\|f\|_{\left.H^{1 /(1+\epsilon)} / \lambda\right)^{1 /(1+\epsilon)},}\right.
\end{aligned}
$$

which together with a density argument completes the proof of Theorem 4.3.

\section{Application to Monge-Ampère Singular Integrals}

In this section, we consider Monge-Ampère singular integral operators introduced by Caffarelli and Gutiérrez in [8], which are related to the real analysis of the Monge-Ampère equation developed in [7]. Let $\mu$ be the Monge-Ampère measure and $d$ be the quasi-metric introduced by Aimar, Forzani and Toledano [1], which 
is related to sections. Then $\left(\mathbb{R}^{m}, d, \mu\right)$ is a space of homogeneous type as pointed out in [1]. We first observe that based on some results in [1], it is easy to see that $H^{1}\left(\mathbb{R}^{m}, d, \mu\right)=H_{\mathcal{F}}^{1}\left(\mathbb{R}^{m}, \mu\right)$, where $H_{\mathcal{F}}^{1}\left(\mathbb{R}^{m}, \mu\right)$ was recently introduced by Ding and Lin [14]. This observation together with Theorem A and Theorem B in [12] immediately implies Theorem 1.1 and Theorem 1.2 in [14]; see Remark 5.1 below. We then verify that the quasi-metric $d$ is equivalent to the "metric" $\widetilde{d}$ introduced by Incognito [21]; see Lemma 5.1 below. From this fact and an observation in [12, p. 599], we immediately deduce the boundedness of Monge-Ampère singular integral operators from $H^{1}\left(\mathbb{R}^{m}, d, \mu\right)$ to $L^{1}\left(\mathbb{R}^{m}, d, \mu\right)$, which was also obtained in [14] by a slight different method. Moreover, in Lemma 5.2 below, we further verify that the kernels of Monge-Ampere singular integral operators satisfy the standard pointwise regularity conditions of Calderon-Zygmund operators, which improves the main result in [21] (see Lemma 1 there) and is useful in applications. Using Theorem 4.1 through Theorem 4.3, we obtain in Proposition 5.1 below the boundedness of Monge-Ampère singular integral operators from $H^{p}\left(\mathbb{R}^{m}, d, \mu\right)$ to $L^{p}\left(\mathbb{R}^{m}, d, \mu\right)$ and from $H^{p}\left(\mathbb{R}^{m}, d, \mu\right)$ to $H^{p}\left(\mathbb{R}^{m}, d, \mu\right)$ with $p \in\left(p_{0}, 1\right]$, and the boundedness from $H^{p_{0}}\left(\mathbb{R}^{m}, d, \mu\right)$ to weak- $L^{p_{0}}\left(\mathbb{R}^{m}, d, \mu\right)$. We now recall some definitions and notation.

For $x \in \mathbb{R}^{m}$ and $t>0$, denote by $S(x, t)$ certain open and bounded convex set containing $x$. We call $\mathcal{F}=\left\{S(x, t): x \in \mathbb{R}^{m}, t>0\right\}$ a family of sections if $\left\{S(x, t): x \in \mathbb{R}^{m}, t>0\right\}$ is monotone increasing in $t$, i. e., $S(x, t) \subset S\left(x, t^{\prime}\right)$ for $t \leq t^{\prime}$, and satisfies the following three conditions:

(A) There exist positive constants $K_{1}, K_{2}$ and $K_{3}$ and $\epsilon_{1}, \epsilon_{2}$ such that given two sections $S\left(x_{0}, t_{0}\right)$ and $S(x, t)$ with $t \leq t_{0}$ satisfying $S\left(x_{0}, t_{0}\right) \cap S(x, t) \neq$ $\emptyset$, and given $T$ an affine transformation that normalizes $S\left(x_{0}, t_{0}\right)$, i. e., $B(0,1 / n) \subset T\left(S\left(x_{0}, t_{0}\right)\right) \subset B(0,1)$, there exists $z \in B\left(0, K_{3}\right)$ depending on $S\left(x_{0}, t_{0}\right)$ and $S(x, t)$ such that $B\left(z, K_{2}\left(t / t_{0}\right)^{\epsilon_{2}}\right) \subset T(S(x, t)) \subset$ $B\left(z, K_{1}\left(t / t_{0}\right)^{\epsilon_{1}}\right)$ and $T(z) \in B\left(z, \frac{1}{2} K_{2}\left(\frac{t}{t_{0}}\right)^{\epsilon_{2}}\right)$. Here and in what follows, $B(x, t)$ denotes the Euclidean open ball centered at the point $x$ with radius $t$.

(B) There exists a constant $\sigma>0$ such that for any given section $S(x, t)$ and $y \notin$ $S(x, t)$, if $T$ is an affine transformation that normalizes $S(x, t), B\left(T(y), \epsilon^{\sigma}\right) \cap$ $T(S(x,(1-\epsilon) t))=\emptyset$ for any $\epsilon \in(0,1)$.

(C) $\cap_{t>0} S(x, t)=\{x\}$ and $\cup_{t>0} S(x, t)=\mathbb{R}^{m}$.

In addition we assume that a Borel regular measure $\mu$ which is finite on compact sets is given, $\mu\left(\mathbb{R}^{m}\right)=\infty$, and satisfies the following doubling condition

$$
\mu(S(x, 2 t)) \leq C_{10} \mu(S(x, t)),
$$

where $C_{10}>0$ is independent of $x$ and $t$. Thus, we know that there exist constants 
$C_{11}>0$ and $n>0$ such that for any $\lambda>1, x \in \mathbb{R}^{m}$ and $t>0$,

$$
\mu(S(x, \lambda t)) \leq C_{11} \lambda^{n} \mu(S(x, t)) .
$$

An important example of family of sections comes from the Monge-Ampere equation which can be given as follows. Let $\varphi: \mathbb{R}^{m} \rightarrow \mathbb{R}$ be a convex smooth function. For any fixed $x \in \mathbb{R}^{m}$, let $\mathcal{L}(x)$ be the supporting hyperplane of $\varphi$ at $(x, \varphi(x))$. For $t>0$, define the set $S_{\varphi}(x, t)=\left\{y \in \mathbb{R}^{m}: \varphi(y)<\mathcal{L}(x)+t\right\}$. Then $\left\{S_{\varphi}(x, t): \quad x \in \mathbb{R}^{m}, t>0\right\}$ is a family of sections that has the above properties (A), (B) and (C). Furthermore, if the graph of $\varphi$ contains no lines, then the Monge-Ampère measure $\mu$ generated by the function $\varphi, \operatorname{det} D^{2} \varphi=\mu$, satisfies the doubling condition (5.1); see $[6,7]$.

The definition of sections was introduced by Caffarelli and Gutierrez [7] to study the real variable theory associated to the Monge-Ampere equation. Caffarelli and Gutiérrez [7] established a Besicovitch type covering lemma for $\mathcal{F}$, a family of sections. In terms of sections, they set up a variant of the Calderon-Zygmund decomposition by applying this covering lemma. As applications of this decomposition, Caffarelli and Gutiérrez introduced the Hardy-Littllewood maximal operator and the space $\mathrm{BMO}_{\mathcal{F}}$ associated to a family of sections and the doubling measure, and obtained some important results. Recently, there are several papers concerning the real analysis associated to the Monge-Ampère equation. Aimar, Forzani and Toledano [1] proved that the properties (A) and (B) imply the following engulfing properties of sections: there is a constant $\delta>1$, depending only on $\sigma, K_{1}$, and $\epsilon_{1}$, such that for any $x, y \in \mathbb{R}^{m}$ and $t>0, y \in S(x, t)$ implies that

$$
S(y, t) \subset S(x, \delta t) \text { and } S(x, t) \subset S(y, \delta t) .
$$

Moreover, they introduced the function

$$
d(x, y)=\inf \{t>0: x \in S(y, t) \text { and } y \in S(x, t)\}
$$

and proved that $d$ is a quasi-metric satisfying that for all $x, y, z \in \mathbb{R}^{m}, d(x, y) \leq$ $\delta(d(x, z)+d(z, y))$, and also that for any $x \in \mathbb{R}^{m}$ and $t>0$,

$$
S(x, t /(2 \delta)) \subset B_{d}(x, t) \subset S(x, t),
$$

where $B_{d}(x, t)=\left\{y \in \mathbb{R}^{m}: d(x, y)<t\right\}$. From this and (5.1), it is easy to deduce that for any $x \in \mathbb{R}^{m}$ and $t>0$,

$$
\mu\left(B_{d}(x, 2 t)\right) \leq\left(C_{10}\right)^{\left[\log _{2}(4 \delta)\right]+1} \mu\left(B_{d}(x, t)\right),
$$

where $\left[\log _{2}(4 \delta)\right]$ is the largest integer no more than $\log _{2}(4 \delta)$; and from this and (5.2), it follows that for any $x \in \mathbb{R}^{m}, t>0$ and $\lambda>1$,

$$
\mu\left(B_{d}(x, \lambda t)\right) \leq C_{11}(2 \delta)^{n} \lambda^{n} \mu\left(B_{d}(x, t)\right) .
$$


Thus $\left(\mathbb{R}^{m}, d, \mu\right)$ is a space of homogeneous type in the sense of Coifman and Weiss [11]; see also [1]. Incognito [21] introduced another "metric" $\widetilde{d}$ associated to the sections:

$$
\widetilde{d}(x, y)=\inf \{t>0: y \in S(x, t)\}
$$

and proved that for all $x, y \in \mathbb{R}^{m}, \widetilde{d}(x, y) \leq \delta \widetilde{d}(y, x)$ and for all $x, y, z \in \mathbb{R}^{m}$,

$$
\widetilde{d}(x, y) \leq \delta^{2}(\widetilde{d}(x, z)+\widetilde{d}(z, y)) .
$$

For $x_{0} \in \mathcal{X}$ and $r>0$, in what follows, we let $B_{\widetilde{d}}\left(x_{0}, r\right)=\left\{x \in \mathcal{X}: \widetilde{d}\left(x_{0}, x\right)<\right.$ $r\}$. With the aid of the function $\widetilde{d}$, Incognito [21] proved that the Monge-Ampere singular integral (see definition below) is bounded from $L^{1}\left(\mathbb{R}^{m}, \mu\right)$ to weak- $L^{1}\left(\mathbb{R}^{m}, \mu\right)$. Ding and Lin [14] introduced the atomic Hardy space $H_{\mathcal{F}}^{1}\left(\mathbb{R}^{m}, \mu\right)$ associated to the sections, and proved that $\mathrm{BMO}_{\mathcal{F}}\left(\mathbb{R}^{m}, \mu\right)$ is the dual space of $H_{\mathcal{F}}^{1}\left(\mathbb{R}^{m}, \mu\right)$, and that the Monge-Ampère singular integral operator is bounded from $H_{\mathcal{F}}^{1}\left(\mathbb{R}^{m}, \mu\right)$ to $L^{1}\left(\mathbb{R}^{m}, \mu\right)$.

For the space of homogeneous type, $\left(\mathbb{R}^{m}, d, \mu\right)$, we denote the atomic Hardy spaces of Coifman and Weiss in [12] (see Definition 2.4 of Section 2) by $H^{p}\left(\mathbb{R}^{m}, d, \mu\right)$ for $0<p \leq 1$.

Remark 5.1. We point out that from the relation (5.5) between balls related to quasi-metric $d$ in (5.4) and sections together with the double properties (5.1) and (5.6), it is easy to see that $H_{\mathcal{F}}^{1}\left(\mathbb{R}^{m}, \mu\right)=H^{1}\left(\mathbb{R}^{m}, d, \mu\right)$ with equivalent norms. This observation together with Theorem A and Theorem B in [12] immediately implies Theorem 1.1 and Theorem 1.2 in [14].

We now recall the definition of Monge-Ampère singular integrals in [8]. For each fixed $y \in \mathbb{R}^{m}$ and $j \in \mathbb{Z}$, denote by $S_{j}(y)$ the section $S\left(y, 2^{j}\right)$. Let $\left\{K_{j}\right\}_{j \in \mathbb{Z}}$ be a sequence of functions on $\mathbb{R}^{m} \times \mathbb{R}^{m}$ such that for all $y \in \mathbb{R}^{m}$, supp $K_{j}(\cdot, y) \subset$ $S_{j}(y), \int_{\mathbb{R}^{m}} K_{j}(x, y) d \mu(x)=0, \sup _{j} \int_{\mathbb{R}^{m}}\left|K_{j}(x, y)\right| d \mu(x) \leq C_{12}$, and all still hold with $x$ and $y$ interchanged; if $T$ is an affine transformation that normalizes the section $S_{j}(y)$, then for some constants $C_{13}>0$ and $\alpha \in(0,1]$, and all $y \in \mathbb{R}^{m}$,

$$
\left|K_{j}(u, y)-K_{j}(v, y)\right|+\left|K_{j}(y, u)-K_{j}(y, v)\right| \leq C_{13} \frac{1}{\mu\left(S_{j}(y)\right)}|T u-T v|^{\alpha}
$$

Let

$$
K=\sum_{j=-\infty}^{\infty} K_{j}
$$


The operator defined by

$$
T f(x)=\int_{\mathbb{R}^{m}} K(x, y) f(y) d \mu(y)
$$

is called the Monge-Ampère singular integral. Caffarelli and Gutierrez [8] proved that for $\alpha=1$, the operator $T$ is bounded on $L^{2}\left(\mathbb{R}^{m}, \mu\right)$. Incognito [21] proved that for $0<\alpha \leq 1, T$ is bounded on $L^{2}\left(\mathbb{R}^{m}, \mu\right)$, and also bounded from $L^{1}\left(\mathbb{R}^{m}, \mu\right)$ to weak- $L^{1}\left(\mathbb{R}^{m}, \mu\right)$. Hence $T$ is bounded on $L^{p}(\mathcal{X})$ for $1<p<\infty$. Moreover, Ding and Lin [14] proved that for $0<\alpha \leq 1, T$ is bounded from $H^{1}\left(\mathbb{R}^{m}, d, \mu\right)$ to $L^{1}\left(\mathbb{R}^{m}, \mu\right)$.

The main result of this section can be stated as follows.

Proposition 5.1. Let $\theta \in(0,1)$ be as in (1.5), $n$ as in (5.7), $\epsilon_{1}$ as in (A), $\alpha \in(0,1]$ and $T$ as in (5.11). Then for $p \in[1 /(1+\theta), 1] \cap\left(n /\left(n+\alpha \epsilon_{1}\right), 1\right], T$ can extend to a bounded linear operator from $H^{p}\left(\mathbb{R}^{m}, d, \mu\right)$ to $L^{p}\left(\mathbb{R}^{m}, d, \mu\right)$ and from $H^{p}\left(\mathbb{R}^{m}, d, \mu\right)$ to $H^{p}\left(\mathbb{R}^{m}, d, \mu\right)$; and if $0<\alpha \epsilon_{1} / n \leq \theta$, then $T$ can extend to a bounded linear operator from $H^{n /\left(n+\alpha \epsilon_{1}\right)}\left(\mathbb{R}^{m}, d, \mu\right)$ to weak- $L^{n /\left(n+\alpha \epsilon_{1}\right)}\left(\mathbb{R}^{m}, d, \mu\right)$.

To prove Proposition 5.1, we need the following lemma, which states that the "distance" functions $d$ in (5.4) and $\widetilde{d}$ in (5.8) are actually equivalent.

Lemma 5.1. Let $\delta>1$ be as in (5.3). Then for all $x, y \in \mathbb{R}^{m}, \delta^{-1} d(x, y) \leq$ $\widetilde{d}(x, y) \leq d(x, y)$.

Proof. From the fact that for all $x, y \in \mathbb{R}^{m},\{r: x \in S(y, r), y \in S(x, r)\} \subset$ $\{r: y \in S(x, r)\}$, it is easy to deduce that $\widetilde{d}(x, y) \leq d(x, y)$.

On the other hand, by the engulfing property (5.3) together with the fact that $\delta>1$, we see that

$$
\begin{aligned}
\{t>0: y \in S(x, t)\} & \subset\{t>0: y \in S(x, t), x \in S(y, \delta t)\} \\
& \subset\{t>0: y \in S(x, \delta t), x \in S(y, \delta t)\} .
\end{aligned}
$$

This immediately implies that $\inf \{t>0: y \in S(x, \delta t), x \in S(y, \delta t)\} \leq \widetilde{d}(x, y)$, and so $d(x, y) \leq \delta \widetilde{d}(x, y)$, which completes the proof of Lemma 5.1.

Incognito [21] proved that the kernel $K$ in (5.10) satisfies the Hörmander condition (4.2) with $\rho$ replaced by $\widetilde{d}$. Furthermore, we can verify that the kernel $K$ in (5.10) actually satisfies the following pointwise regularity.

Lemma 5.2. Let $K$ be as in (5.10) with $\alpha \in(0,1]$ and $\epsilon_{1}$ as in (A). Then there exists constants $C_{K}>2 \delta$ and $C>0$ such that for all $x, y, z \in \mathbb{R}^{m}$ with 


$$
\begin{aligned}
& d(x, z) \geq C_{K} d(x, y) \\
& |K(z, x)-K(z, y)|+|K(x, z)-K(y, z)| \leq C\left[\frac{d(x, y)}{d(x, z)}\right]^{\alpha \epsilon_{1}} \frac{1}{\mu\left(B_{d}(x, d(x, z))\right)} .
\end{aligned}
$$

Moreover, there exists a constant $\widetilde{C}>0$ such that $K, K^{*} \in D_{d}(\infty, \eta)$, where $K^{*}(x, y)=K(y, x)$ for all $x, y \in \mathbb{R}^{m}$ with $x \neq y$ and $\eta=\left\{\widetilde{C} 2^{-k \alpha \epsilon_{1} / n}\right\}_{k \in \mathbb{N}}$.

Proof. For fixed $x, y \in \mathbb{R}^{m}$, let $j_{0}$ be an integer such that $2^{j_{0}-1} \leq \widetilde{d}(x, y)<$ $2^{j_{0}}$. Then for all $j \geq j_{0}$ and $z \in S_{j}(y) \cup S_{j}(x)$, Incognito in [21, pp. 44-45] proved that

$$
\left|K_{j}(z, x)-K_{j}(z, y)\right| \lesssim \frac{2^{\alpha \epsilon_{1}\left(j_{0}-j\right)}}{\mu\left(S_{j}(z)\right)}
$$

For any $z \in \mathbb{R}^{m}$ with $\widetilde{d}(x, z) \geq 4 \delta^{3} \widetilde{d}(x, y)$, by (5.9), we have $2 \delta^{-2} \widetilde{d}(x, z) \leq$ $\widetilde{d}(y, z) \leq 2 \widetilde{\delta}^{2} d(x, z)$. Let $k_{0}>j_{0}$ be the largest integer such that $2^{k_{0}-1} \leq$ $(2 \delta)^{-2} \widetilde{d}(x, z)<2^{k_{0}}$. By (5.2) and Lemma 5.1 together (5.7), we have $\mu\left(S_{k_{0}}(z)\right) \sim$ $\mu\left(B_{\widetilde{d}}(z, \widetilde{d}(x, z))\right)$, from which together with (5.12), we deduce that

$$
\begin{aligned}
& |K(z, x)-K(z, y)| \\
& \quad \leq \sum_{j \in \mathbb{Z}}\left|K_{j}(z, x)-K_{j}(z, y)\right| \chi_{S_{j}(x) \cup S_{j}(y)}(z) \\
& \quad \lesssim \sum_{j \geq k_{0}} \frac{2^{\alpha \epsilon_{1}\left(j_{0}-j\right)}}{\mu\left(S_{j}(z)\right)} \lesssim \frac{2^{\alpha \epsilon_{1}\left(j_{0}-k_{0}\right)}}{\mu\left(S_{k_{0}}(z)\right)} \lesssim\left[\frac{\widetilde{d}(x, y)}{\widetilde{d}(z, x)}\right]^{\alpha \epsilon_{1}} \frac{1}{\mu\left(B_{\widetilde{d}}(z, \widetilde{d}(x, z))\right)}
\end{aligned}
$$

Since $K^{*}$ satisfies the same conditions as $K$, the above estimate still holds for $K^{*}$. Thus, from this, Lemma 5.1 and (1.4), it follows the first conclusion of Lemma 5.2. From this together with Definition 1.1, it is easy to verify that $K, K^{*} \in D_{d}(\infty, \eta)$, which completes the proof of Lemma 5.2.

Proof of Proposition 5.1. From Lemma 5.2 and Proposition 4.1, it is easy to deduce that $K \in D_{\rho}\left(\infty, \eta^{(\infty)}\right)$ with $\eta_{k}^{(\infty)}=C 2^{-\alpha \epsilon_{1} k / n}$ for $k \in \mathbb{N}$ and certain constant $C>0$. By this and $L^{2}\left(\mathbb{R}^{m}, \mu\right)$-boundedness of $T$ together with the remark in [12, p. 599], it immediately follows that $T$ is bounded from $H^{1}\left(\mathbb{R}^{m}, d, \mu\right)$ to $L^{1}\left(\mathbb{R}^{m}, d, \mu\right)$. Moreover, applying Theorem 4.1 through Theorem 4.3, we obtain all the conclusions in Proposition 5.1, which completes the proof of Proposition 5.1.

Remark 5.2. The boundedness of $T$ from $H^{1}\left(\mathbb{R}^{m}, d, \mu\right)$ to $L^{1}\left(\mathbb{R}^{m}, d, \mu\right)$ was also proved in [14] by a slight different method; see Remark 5.1. 
Remark 5.3. By the proof of Theorem 2 in [23], we know that $\theta$ in (1.5) can be taken to be $1 / \log _{2}\left[C_{4}\left(2 C_{4}+1\right)\right]$. It will be interesting to find the biggest $\theta$ which guarantees (1.5).

\section{ACKNOWLEDGMENT}

The authors would like to thank Professors Yongsheng Han and Marcin Bownik for some helpful discussions on some parts of this paper.

\section{REFERENCES}

1. H. Aimar, L. Forzani and R. Toledano, Balls and quasi-metrics: a space of homogeneous type modeling the real analysis related to the Monge-Ampere equation, $J$. Fourier Anal. Appl., 4 (1998), 377-381.

2. J. Alvarez, $H^{p}$ and weak $H^{p}$ continuity of Calderón-Zygmund type operators, in: Fourier analysis (Orono, ME, 1992), Lecture Notes in Pure and Appl. Math., 157, Dekker, New York, 1994, pp. 17-34.

3. M. Bownik, Boundedness of operators on Hardy spaces via atomic decompositions, Proc. Amer. Math. Soc., 133 (2005), 3535-3542.

4. M. Bownik, Anisotropic Hardy spaces and wavelets, Mem. Amer. Math. Soc., 164 (2003), 1-122.

5. A. Baernstein II and E. T. Sawyer, Embedding and multiplier theorems for $H^{p}\left(R^{n}\right)$, Mem. Amer. Math. Soc., 53 (1985), 1-82.

6. L. A. Caffarelli, Interior a priori estimates for solutions of fully nonlinear equations, Ann. of Math. (2), 130 (1989), 189-213.

7. L. A. Caffarelli and C. E. Gutiérrez, Real analysis related to the Monge-Ampère equation, Trans. Amer. Math. Soc., 348 (1996), 1075-1092.

8. L. A. Caffarelli and C. E. Gutiérrez, Singular integrals related to the Monge-Ampère equation, in: Wavelet theory and harmonic analysis in applied sciences (Buenos Aires, 1995), Appl. Numer. Harmon. Anal., Birkhäuser Boston, Boston, MA, 1997, pp. 3-13.

9. R. R. Coifman, A real variable characterization of $H^{p}$, Studia Math., 51 (1974), 269-274.

10. R. R. Coifman, P.-L. Lions, Y. Meyer and S. Semmes, Compensated compactness and Hardy spaces, J. Math. Pures Appl. (9), 72 (1993), 247-286.

11. R. R. Coifman et G. Weiss, Analyse harmonique non-commutative sur certains espaces homogènes, Lecture Notes in Math., Vol. 242, Springer, Berlin, 1972.

12. R. R. Coifman and G. Weiss, Extensions of Hardy spaces and their use in analysis, Bull. Amer. Math. Soc., 83 (1977), 569-645. 
13. G. David, J.-L. Journé et S. Semmes, Opérateurs de Calderón-Zygmund, fonctions para-accrétives et interpolation, Rev. Mat. Ibero., 1 (1985), 1-56.

14. Y. Ding and C. Lin, Hardy spaces associated to the sections, Tohoku Math. J. (2), 57 (2005), 147-170.

15. A. E. Gatto and S. Vági, On molecules and fractional integrals on spaces of homogeneous type with finite measure, Studia Math., 103 (1992), 25-39.

16. J. García-Cuerva and J. L. Rubio de Francia, Weighted Norm Inequalities and Related Topics, North-Holland, Amsterdam, 1985.

17. C. Fefferman and E. M. Stein, $H^{p}$ spaces of several variables, Acta Math., 129 (1972), 137-193.

18. Y. Han, Triebel-Lizorkin spaces on spaces of homogeneous type, Studia Math., 108 (1994), 247-273.

19. Y. Han, D. Müller and D. Yang, Littlewood-Paley characterizations for Hardy spaces on spaces of homogeneous type, Math. Nachr., 279 (2006), 1505-1537.

20. J. Heinonen, Lectures on Analysis on Metric Spaces, Springer-Verlag, New York, 2001.

21. A. Incognito, Weak-type $(1,1)$ inequality for the Monge-Ampere SIO's, J. Fourier Anal. Appl., 7 (2001), 41-48.

22. R. H. Latter, A characterization of $H^{p}\left(\mathbb{R}^{n}\right)$ in terms of atoms, Studia Math., 62 (1978), 93-101.

23. R. A. Macías and C. Segovia, Lipschitz functions on spaces of homogeneous type, Adv. in Math., 33 (1979), 257-270.

24. R. A. Macías and C. Segovia, A decomposition into atoms of distributions on spaces of homogeneous type, Adv. in Math., 33 (1979), 271-309.

25. R. A. Macías and C. Segovia, Singular integrals on generalized Lipschitz and Hardy spaces, Studia Math., 65 (1979), 55-75.

26. Y. Meyer and R. R. Coifman, Wavelets. Calderón-Zygmund and Multilinear Operators, Cambridge University Press, Cambridge, 1997.

27. Y. Meyer, M. Taibleson and G. Weiss, Some functional analytic properties of the spaces $B_{q}$ generated by blocks, Indiana Univ. Math. J., 34 (1985), 493-515.

28. S. Müller, Hardy space methods for nonlinear partial differential equations, Tatra Mt. Math. Publ., 4 (1994), 159-168.

29. J. L. Rubio de Francia, F. J. Ruiz and J. L. Torrea, Calderón-Zygmund theory for operator-valued kernels, Adv. in Math., 62 (1986), 7-48.

30. S. Semmes, A primer on Hardy spaces, and some remarks on a theorem of Evans and Müller, Comm. Partial Differential Equations, 19 (1994), 277-319.

31. E. M. Stein, Singular Integrals and Differentiability Properties of Functions, Princeton University Press, Princeton, N. J., 1970. 
32. E. M. Stein, Harmonic Analysis: Real-variable Methods, Orthogonality, and Oscillatory Integrals, Princeton University Press, Princeton, N. J., 1993.

33. E. M. Stein, M. H. Taibleson and G. Weiss, Weak type estimates for maximal operators on certain $H^{p}$ classes, Rend. Circ. Mat. Palermo (2) Suppl., 1 (1981), 8197.

34. E. M. Stein and G. Weiss, On the theory of harmonic functions of several variables. I. The theory of $H^{p}$-spaces, Acta Math., 103 (1960), 25-62.

35. M. H. Taibleson and G. Weiss, The molecular characterization of certain Hardy spaces, Astérisque, 77 (1980), 67-149.

36. K. Yabuta, A remark on the $\left(H^{1}, L^{1}\right)$ boundedness, Bull. Fac. Sci. Ibaraki Univ. Ser. A, 25 (1993), 19-21.

\section{Guoen $\mathrm{Hu}$}

Department of Applied Mathematics,

University of Information Engineering,

P. O. Box 1001-747,

Zhengzhou 450002,

P. R. China

E-mail: huguoen@eyou.com

Dachun Yang and Yuan Zhou

School of Mathematical Sciences,

Beijing Normal University,

Laboratory of Mathematics and Complex Systems,

Ministry of Education,

Beijing 100875,

P. R. China

E-mail: dcyang@bnu.edu.cn yuanzhou@mail.bnu.edu.cn 